\title{
Randomly repeated measurements on quantum systems: Correlations and topological invariants of the quantum evolution
}

\author{
K. Ziegler ${ }^{1}$, E. Barkai ${ }^{2}$ and D. Kessler ${ }^{3}$ \\ ${ }^{1}$ Institut für Physik, Universität Augsburg, D-86135 Augsburg, Germany \\ ${ }^{2}$ Department of Physics, Institute of Nanotechnology and Advanced Materials, \\ Bar-Ilan University, Ramat Gan 52900, Israel \\ ${ }^{3}$ Department of Physics, Bar-Ilan University, Ramat Gan 52900, Israel
}

(Dated: June 11, 2021)

\begin{abstract}
Randomly repeated measurements during the evolution of a closed quantum system create a sequence of probabilities for the first detection of a certain quantum state. The related discrete monitored evolution for the return of the quantum system to its initial state is investigated. We found that the mean number of measurements until the first detection is an integer, namely the dimensionality of the accessible Hilbert space. Moreover, the mean first detected return time is equal to the average time step between successive measurements times the mean number of measurements. Thus, the mean first detected return time scales linearly with the dimensionality of the accessible Hilbert space. The main goal of this work is to explain the quantization of the mean return time in terms of a quantized Berry phase.
\end{abstract}

\section{INTRODUCTION}

The unitary evolution (UE) of a closed quantum system from the initial state $|\Psi\rangle$ to the state $|\Psi(\tau)\rangle$ on the time interval $\tau$ is defined by $|\Psi(\tau)\rangle=\exp (-i H \tau / \hbar)|\Psi\rangle$, where $H$ is the Hamiltonian of the system. The result of this evolution is characterized by the overlap amplitude $\langle\psi \mid \Psi(\tau)\rangle$ with respect to a given state $|\psi\rangle$. Then $|\langle\psi \mid \Psi(\tau)\rangle|^{2}$ is the probability that the evolution has reached the state $|\psi\rangle$, which obviously depends on the time $\tau$. In a single experiment the measurement time is fixed and, therefore, the UE allows us to detect the state $|\psi\rangle$ with probability $|\langle\psi \mid \Psi(\tau)\rangle|^{2}$ only once. The detection of this state at different times would require the repetition of the experiment, prepared in the same initial state $|\Psi\rangle$, for different values of $\tau[1]$. An alternative approach, which we call "monitored evolution (ME)" is to allow the system to evolve from the initial state $|\Psi\rangle$ for the time $\tau_{1}$ and then measure whether or not the system is in the state $|\psi\rangle$. If the answer is "yes", we stop the experiment, if the answer is "no" we allow the system to evolve further after the measurement [2] and perform a second measurement at time $\tau_{1}+\tau_{2}$. This procedure is repeated for times $t_{2}=\tau_{1}+\tau_{2}, \ldots, t_{k}=\tau_{1}+\cdots+\tau_{k}$ until the measurement detects the state $|\psi\rangle$ for the first time. We consider cases in which the state is detected with probability one. This is called a recurrent measurement process [1]. If at time $t_{1}$ the outcome of the measurement is null this can be associated with a projection of the quantum system $(\mathbf{1}-|\psi\rangle\langle\psi|)\left|\Psi\left(t_{1}\right)\right\rangle$ with a subsequent normalization of the resulting state [3], and similarly for $t_{2}$ etc. And when the state is detected for the first time at time $t_{k}$, the amplitude of the corresponding state is $\phi_{k}$ and its probability is $\left|\phi_{k}\right|^{2}$ [4, 5].

For fixed time steps $\tau$ with $t_{k}=k \tau$ the repeated measurement approach, also known as the stroboscopic protocol, has been studied in great detail in Refs. [1] and [3 19]. Two cases have been distinguished, the return probability for $|\psi\rangle=|\Psi\rangle$ and the transition probability for $|\psi\rangle \neq|\Psi\rangle$. It was found that (i) the return and the transition probabilities differ qualitatively, (ii) the average first detected return time is quantized and given by the winding number of the Laplace transform of the return amplitude [1], (iii) near degeneracies of the spectrum of the evolution operator, the fluctuations become very large and diverge at the degeneracies [3, 11, 18], and (iv) the average first detected transition time also diverges at the degeneracies [20].

Our main intention is to present in this article a study of the effect of independent and identically distributed random time steps $\left\{\tau_{k}\right\}$ on the ME. For this purpose we want to answer the following questions: (1) Do we still obtain quantization of the number of attempts for the first successful measurement? (2) Is the average mean time for the first detection also quantized, as found for stroboscopic measurements? (3) How can dynamical quantization be related to topological invariants? (4) Do random time steps affect the divergent fluctuations near resonances? To answer these questions we will develop a theory of the first detection time under repeated random measurements. Our work is based on the ideas presented by Grünbaum et al. 9] for fixed time steps and related to a work by Varbanov et al. 21] on random time steps, who studied the conditions for the existence of non-detectable dark states. Random time measurements were discussed also for open quantum systems 22], while for closed quantum systems they 
were recently studied by us in terms of average return and transition probabilities [? ]. The present work is an extension of the latter in which we explain in detail the origin of the quantization of the mean return time. For this purpose we compare the Berry phase of the return amplitude, averaged with respect to the distribution of the measurement times. It will be shown that the average Berry phase is equal to the average number of measurements for the first detected return and equal to the dimensionality of the accessible Hilbert space. The average Berry phase is reminiscent of the quantized winding number in case of stroboscopic measurements [9].

The structure of this article is as follows. In Sect. @ the definitions of the relevant quantities for the first detection under repeated measurements are given. Then a short summary of the main results are presented in Sect. III] After a brief discussion of the ME for fixed time steps $\tau$ in Sect. IV] we formulate the ME for random time steps in terms of random matrix products in Sects. VIVIB 1] This includes a description of the averaging procedure (Sect. VI) and the introduction of a generating function for the average return time and its higher moments (Sect. VIA). The quantization of the mean number of measurements of the first detected return by the dimensionality of the accessible Hilbert space is studied in Sects. VIB andVIB 1] Finally, in Sect. VII the example of a symmetric two-level system is analyzed, followed by a discussion of all the results in Sect. VIII Details of the calculations are presented in Apps. A. F .

\section{RETURN AMPLITUDE OF THE MONITORED EVOLUTION}

The return probability as a function of time, with or without intermediate measurements, provides a measure of how big the accessible space is and how long it takes to return to the initial state. This is an important quantity for classical random walks 23, 24] and plays also an important role for characterizing localization in many-body quantum systems [25, 26]. We will investigate the return amplitude for the ME with measurements at time steps $\left\{\tau_{k}\right\}_{k=1,2, \ldots}$. First, we will have need to refer to the return amplitude of the UE

$$
u_{k}=\left\langle\Psi\left|e^{-i H\left(\tau_{1}+\cdots+\tau_{k}\right)}\right| \Psi\right\rangle
$$

for the state $|\Psi\rangle$ when we measure only once after the time $t_{k}=\tau_{1}+\cdots+\tau_{k}$, assuming that the UE is governed by the Hamiltonian $H$. Then we turn to the return amplitude for the ME [3, 4, 13]

$$
\phi_{k}=\left\langle\Psi\left|e^{-i H \tau_{k}}\left(P e^{-i H \tau_{k-1}}\right) \cdots\left(P e^{-i H \tau_{1}}\right)\right| \Psi\right\rangle, \quad P=\mathbf{1}-|\Psi\rangle\langle\Psi|,
$$

which is the major objective of our inquiry. This is the return amplitude at time $t_{k}$, provided that we also measure at times $t_{1}, t_{2}, \ldots, t_{k-1}$ but detect the quantum state $|\Psi\rangle$ for the first time at $t_{k}$ with probability $\left|\phi_{k}\right|^{2}$. For the time of measurements we assume a distribution density $\mathcal{P}\left(\left\{\tau_{k}\right\}\right)$ of independent and identically distributed time steps, such that $\mathcal{P}\left(\left\{\tau_{k}\right\}\right)=\prod_{k} P\left(\tau_{k}\right)$. This can be understood as the effect of an inaccurate clock. This enables us to consider the average $\langle\ldots\rangle_{\tau} \equiv \int \ldots \prod_{k} \mathcal{P}\left(\tau_{k}\right) d \tau_{k}$ with respect to the ensemble of random time steps. Here we do not specify the distribution. For instance, $\mathcal{P}\left(\tau_{k}\right)$ can be a Dirac delta, which would recover the stroboscopic ME [3, 9, 13, 18], it could be an exponential distribution or any other distribution, which allows us to perform the average $\langle\ldots\rangle_{\tau}$.

Besides the averaging with respect to times steps $\left\{\tau_{k}\right\}$ we also need to define the averages

$$
\overline{k^{m}}:=\sum_{k \geq 1} k^{m}\left\langle\left|\phi_{k}\right|^{2}\right\rangle_{\tau}, \quad \overline{t^{m}}:=\sum_{k \geq 1}\left\langle t_{k}^{m}\left|\phi_{k}\right|^{2}\right\rangle_{\tau} \quad(m=1,2, \ldots) .
$$

Here it is assumed that $\sum_{k}\left|\phi_{k}\right|^{2}=1$, which is justified for a finite-dimensional Hilbert space (cf. App. A). This equation means that the state is eventually detected [9]. Thus, the overline represents a double average, namely an average with respect to the number of time steps $k=1,2, \ldots$ with weight $\left|\phi_{k}\right|^{2}$, followed by the average $\langle\ldots\rangle_{\tau}$. For $m=1$ both expressions in Eq. (3) will be used to characterize the $\mathrm{ME}: \bar{k}$ is the mean number of measurements for the first detected return (FDR) and $\bar{t}$ is the mean FDR time.

Since both evolutions are defined on an $N$-dimensional Hilbert space by the Hamiltonian $H$, we consider its eigenstates $\left\{\left|E_{j}\right\rangle\right\}_{j=1, \ldots, N}$ and its corresponding eigenvalues $\left\{E_{j}\right\}_{j=1, \ldots, N}$. Then the return amplitude 
$\phi_{k}$ of the ME can be expressed as a matrix product, which is efficiently written in the energy representation as a sum over all energy levels $\left\{E_{j}\right\}_{j=1,2, \ldots, N}$ as

$$
\phi_{k}=\sum_{j_{1}, j_{2}, \ldots, j_{k}=1}^{N}\left\langle\Psi \mid E_{j_{k}}\right\rangle e^{-i E_{j_{k}} \tau_{k}}\left\langle E_{j_{k}}|P| E_{j_{k-1}}\right\rangle e^{-i E_{j_{k-1}} \tau_{k-1}} \cdots\left\langle E_{j_{2}}|P| E_{j_{1}}\right\rangle e^{-i E_{j_{1}} \tau_{1}}\left\langle E_{j_{1}} \mid \Psi\right\rangle .
$$

In principle, there is the possibility of degenerate eigenvalues or of a vanishing overlap $p_{j}=\left|\left\langle E_{j} \mid \Psi\right\rangle\right|^{2}$, which must be treated with care [9]. To understand the effect of degenerate energy levels on $\phi_{k}$ we consider the levels $E_{j}, E_{j^{\prime}}$ and assume at first that they are not degenerate. Then we can write

$$
\begin{gathered}
\sum_{j_{k}=1}^{N}\left\langle E_{j_{k-1}}|P| E_{j_{k}}\right\rangle e^{-i E_{j_{k}} \tau_{k}}\left\langle E_{j_{k}}|P| E_{j_{k+1}}\right\rangle=\sum_{j_{k}=1, j_{k} \neq j, j^{\prime}}^{N} e^{-i E_{j_{k}} \tau_{k}}\left\langle E_{j_{k-1}}|P| E_{j_{k}}\right\rangle\left\langle E_{j_{k}}|P| E_{j_{k+1}}\right\rangle \\
+\left\langle E_{j_{k-1}}\left|P\left[e^{-i E_{j} \tau_{k}}\left|E_{j}\right\rangle\left\langle E_{j}\left|+e^{-i E_{j^{\prime}} \tau_{k}}\right| E_{j^{\prime}}\right\rangle\left\langle E_{j^{\prime}}\right|\right] P\right| E_{j_{k+1}}\right\rangle,
\end{gathered}
$$

where the second term on the right-hand side describes the evolution of the state $P\left|E_{j_{k+1}}\right\rangle$ with the evolution operator

$$
P\left[e^{-i E_{j} \tau_{k}}\left|E_{j}\right\rangle\left\langle E_{j}\left|+e^{-i E_{j^{\prime}} \tau_{k}}\right| E_{j^{\prime}}\right\rangle\left\langle E_{j^{\prime}}\right|\right]
$$

over the time period $\tau_{k}$. The evolution creates a superposition of the states $\left|E_{j}\right\rangle$ and $\left|E_{j^{\prime}}\right\rangle$, which changes in time due to the time dependent coefficients, provided that $E_{j} \neq E_{j^{\prime}}$. On the other hand, for the degenerate case $E_{j}=E_{j^{\prime}}$, the superposition of $\left|E_{j}\right\rangle$ and $\left|E_{j^{\prime}}\right\rangle$ is fixed during the time period $\tau_{k}$ :

$$
e^{-i E_{j} \tau_{k}} P\left[\left|E_{j}\right\rangle\left\langle E_{j}|+| E_{j^{\prime}}\right\rangle\left\langle E_{j^{\prime}}\right|\right]
$$

and only the global phase changes. This reflects a dimensional reduction of the accessible Hilbert space by 1 , implying that we should use the replacement

$$
\left|E_{j}\right\rangle,\left|E_{j^{\prime}}\right\rangle \rightarrow\left|E_{j j^{\prime}}\right\rangle:=\left|E_{j}\right\rangle\left\langle E_{j} \mid \Psi\right\rangle+\left|E_{j^{\prime}}\right\rangle\left\langle E_{j^{\prime}} \mid \Psi\right\rangle
$$

and the simultaneous elimination of $j^{\prime}$ from the summation of $j_{k}$ in Eq. (4).

Another special case is a vanishing overlap $p_{j}=\left|\left\langle E_{j} \mid \Psi\right\rangle\right|^{2}=0$. Beginning with the initial state on the right-hand side of Eq. (4) we get

$$
\sum_{j_{1}=1}^{N}\left\langle E_{j_{2}}|P| E_{j_{1}}\right\rangle e^{-i E_{j_{1}} \tau_{1}}\left\langle E_{j_{1}} \mid \Psi\right\rangle=\sum_{j_{1}=1 ; j_{1} \neq j}^{N}\left\langle E_{j_{2}}|P| E_{j_{1}}\right\rangle e^{-i E_{j_{1}} \tau_{1}}\left\langle E_{j_{1}} \mid \Psi\right\rangle .
$$

Next, in the summation with respect to $j_{2}$ the special value $j$ does not contribute again, since $j_{2}=j$ gives

$$
\left\langle E_{j}|P| E_{j_{1}}\right\rangle=\left\langle E_{j} \mid E_{j_{1}}\right\rangle-\left\langle E_{j} \mid \Psi\right\rangle\left\langle\Psi \mid E_{j_{1}}\right\rangle=0
$$

due to $j_{1} \neq j$ and due to $\left\langle E_{j} \mid \Psi\right\rangle=0$. Repeating this argument we find that the value $j$ drops out of all summations in Eq. (4), reducing the accessible Hilbert space by 1 again.

With these arguments we have removed the degeneracy of the energy levels and the vanishing overlaps $p_{j}$. Therefore, the remaining return amplitude $\phi_{k}$ depends only non-degenerate energy levels and on overlaps with $p_{j}>0$. For the subsequent analysis we use the convention that $N$ is the dimensionality of the accessible Hilbert space.

For the subsequent calculations it is useful to introduce two types of discrete Fourier transformations, where one is based on the phase factors $e^{i \omega k}$

$$
\tilde{\phi}(\omega)=\sum_{k \geq 1} e^{i \omega k} \phi_{k}
$$

and the other is based on the random phase factors $e^{i \omega\left(\tau_{1}+\cdots+\tau_{k}\right)} \equiv e^{i \omega t_{k}}$

$$
\tilde{\phi}_{\tau}(\omega)=\sum_{k \geq 1} e^{i \omega t_{k}} \phi_{k}
$$

provided that these series exist. Both $\tilde{\phi}(\omega)$ and $\tilde{\phi}_{\tau}(\omega)$ are still functions of the random variables $\left\{\tau_{k}\right\}$. 


\section{SUMMARY AND RESULTS}

It will be shown that for a quantum system with energy levels $\left\{E_{j}\right\}$ and eigenstates $\left\{\left|E_{j}\right\rangle\right\}$ of a given Hamiltonian $H$ the FDR probability $\left|\phi_{k}\right|^{2}$ in the case of a ME is determined by the random phase factors $\left\{e^{-i E_{j} \tau_{k}}\right\}$ and the overlaps $\left\{\left|\left\langle E_{j} \mid \Psi\right\rangle\right|^{2}\right\} \equiv\left\{p_{j}\right\}$ alone. After averaging with respect to the random measurements we get $\left\langle\left|\phi_{k}\right|^{2}\right\rangle_{\tau}$, which will turn out to be a function of the $N$ parameters $\left\{\left\langle e^{-i E_{j} \tau}\right\rangle_{\tau}\right\}$ and of the $N^{2}$ parameters $\left\{\left\langle e^{-i\left(E_{j}-E_{j^{\prime}}\right) \tau}\right\rangle_{\tau}\right\}$. (Details are given in Sect. V) Using the Fourier transformations (5) and (6), we define the generating functions $F(\omega)=\sum_{k>1} e^{i k \omega}\left\langle\left|\phi_{k}\right|^{2}\right\rangle_{\tau}$ and $F_{\tau}(\omega)=\sum_{k>1}\left\langle e^{i \omega t_{k}}\left|\phi_{k}\right|^{2}\right\rangle_{\tau}$. By differentation with respect to $\omega$ we get the mean number of measurements for the FDR and the mean FDR time as

$$
\bar{k}=-\left.i \partial_{\omega} F(\omega)\right|_{\omega=0}, \quad \bar{t}=-\left.i \partial_{\omega} F_{\tau}(\omega)\right|_{\omega=0} .
$$

Then we will derive the relation $\bar{t}=\langle\tau\rangle_{\tau} \bar{k}$, where $\langle\tau\rangle_{\tau}$ is the mean time interval between successive measurements.

Besides the average FDR probability $\left\langle\left|\phi_{k}\right|^{2}\right\rangle_{\tau}$ we will also calculate the corresponding expressions of the Fourier transform $\tilde{\phi}(\omega)$, namely $\left\langle|\tilde{\phi}(\omega)|^{2}\right\rangle_{\tau}$. This will turn out to be 1 , as shown in Eq. (33), which is essential for calculating the average Berry phase and the mean values of the FDR as

$$
\bar{k}=N, \bar{t}=\langle\tau\rangle_{\tau} N .
$$

The main result are listed in Table \.

\section{FIXED TIME STEP $\tau$}

In this section we briefly recapitulate what is known about the FDR problem in the case of nonrandom $\tau$ to set the stage for our investigation of random time steps $\left\{\tau_{k}\right\}$. Stroboscopic measurement with $\tau_{k}=\tau$ has been studied extensively in the literature [3, 9, 11, 13, 14, 18]. Next we summarize relevant information from previous works, in particular, some results of Ref. [18].

At fixed $\tau$ the Laplace transformation for the return amplitude of the ME reads

$$
\hat{\phi}(z) \equiv \sum_{k \geq 1} z^{k} \phi_{k}=\sum_{k \geq 1} z^{k}\left\langle\Psi\left|\left(e^{-i H \tau} P\right)^{k-1} e^{-i H \tau}\right| \Psi\right\rangle=\left\langle\Psi\left|\left(e^{i \tau H} / z-P\right)^{-1}\right| \Psi\right\rangle .
$$

Due to the relation of Eq. (B3) in App. B, we obtain for $K=e^{i \tau H} / z-\mathbf{1}$ the following identity

$$
\left\langle\Psi\left|(K+|\Psi\rangle\langle\Psi|)^{-1}\right| \Psi\right\rangle=1-\frac{1}{1+\left\langle\Psi\left|K^{-1}\right| \Psi\right\rangle} .
$$

This identity is important because it allows us to represent the projector-dependent left-hand side by the expression $\left\langle\Psi\left|K^{-1}\right| \Psi\right\rangle$, which is diagonal in terms of the energy eigenstates of $H$ and independent of the projector $|\Psi\rangle\langle\Psi|$. A corresponding identity exists in the case of random time steps, which will be central for our subsequent calculations.

With $u_{k}$ of Eq. (11) we can write $\left\langle\Psi\left|K^{-1}\right| \Psi\right\rangle$ as the Laplace transform of $u_{k}:\left\langle\Psi\left|K^{-1}\right| \Psi\right\rangle=\sum_{k \geq 1} z^{k} u_{k} \equiv$ $\hat{u}(z)$. Then $\hat{\phi}(z)$ in Eq. (8), together with Eq. (9), becomes

$$
\hat{\phi}(z)=1-\frac{1}{1+\hat{u}(z)} .
$$

By analytic continuation to the unit circle $z \rightarrow e^{i \omega}$ we get $\hat{u}(z) \rightarrow \tilde{u}(\omega)$ and $\hat{\phi}(z) \rightarrow \tilde{\phi}(\omega)$. Since $\operatorname{Re}[\tilde{u}(\omega)]=-1 / 2$, the expression $\tilde{\phi}$ is unimodular:

$$
\tilde{\phi}(\omega)=\frac{-1 / 2+i \operatorname{Im}[\tilde{u}]}{1 / 2+i \operatorname{Im}[\tilde{u}]}=-\frac{\tilde{u}}{\tilde{u}^{*}},
$$

such that we can write

$$
\tilde{\phi}(\omega)=-e^{2 i \arg [\tilde{u}(\omega)]} \equiv e^{i \varphi(\omega)} .
$$


This result for fixed time steps indicates that the UE and the ME have the same phase change with $\omega$ except for a factor 2. The winding number of $\tilde{\phi}(\omega)$ around the unit circle (i.e. for $0 \leq \omega<2 \pi$ ) is identical with $\sum_{k \geq 1} k\left|\phi_{k}\right|^{2}$, and it is known that the winding number is equal to the dimensionality of the Hilbert space [9].

\section{MATRIX PRODUCTS}

Our goal is to calculate the probability $\left|\phi_{k}\right|^{2}$ of the return amplitude $\phi_{k}$ of Eq. (4) for the general case of random time steps. We would expect that the calculations of the previous section can be extended to this situation. As we will see though it requires some additional steps to calculate quantities, such as the mean FDR time, that are averaged with respect to the random time steps. Our calculation starts with the matrix representation of the projector $P$ of Eq. (2) in terms of energy eigenstates

$$
\left\langle E_{j}|P| E_{j^{\prime}}\right\rangle=\delta_{j, j^{\prime}}-q_{j} q_{j^{\prime}}^{*}, \quad q_{j}=\left\langle E_{j} \mid \Psi\right\rangle,
$$

since the eigenstates are orthonormal: $\left\langle E_{j} \mid E_{j^{\prime}}\right\rangle=\delta_{j, j^{\prime}}$. This is automatically fulfilled for non-degenerate eigenvalues. The above expression is inserted in Eq. (4) and yields for the return amplitude a trace of a matrix product:

$$
\phi_{k}=\operatorname{Tr}\left[D_{k}\left(\mathbf{1}-Q E Q^{*}\right) D_{k-1}\left(\mathbf{1}-Q E Q^{*}\right) \cdots D_{2}\left(\mathbf{1}-Q E Q^{*}\right) D_{1} Q E Q^{*}\right]
$$

with the $N \times N$ matrix $E$, whose elements are all 1 , and with the diagonal matrices $D_{k}=\operatorname{diag}\left(\exp \left(-i E_{1} \tau_{k}\right), \exp \left(-i E_{2} \tau_{k}\right), \ldots, \operatorname{ex}\right.$ and $Q=\operatorname{diag}\left(q_{1}, q_{2}, \ldots, q_{N}\right)$.

Now $Q Q^{*}=\Pi$ is the diagonal matrix $\Pi=\operatorname{diag}\left(p_{1}, p_{2}, \ldots, p_{N}\right)$, which enables us to rewrite $\phi_{k}$ as

$$
\phi_{k}=\operatorname{Tr}\left[D_{k}(\mathbf{1}-E \Pi) D_{k-1}(\mathbf{1}-E \Pi) \cdots D_{2}(\mathbf{1}-E \Pi) D_{1} E \Pi\right],
$$

since $\Pi$ and $D_{j}$ as diagonal matrices commute. This means that $\phi_{k}$ depends only on the spectral weights $\left\{p_{j}\right\}$ through $\Pi$ and on the energy levels $\left\{E_{j}\right\}$ through $D_{k}$.

For the calculation of the return probability we need the product of two traces

$$
\begin{aligned}
& \left|\phi_{k}\right|^{2}=\operatorname{Tr}\left[D_{k}(\mathbf{1}-E \Pi) D_{k-1}(\mathbf{1}-E \Pi) \cdots D_{2}(\mathbf{1}-E \Pi) D_{1} E \Pi\right]^{*} \\
& \quad \times \operatorname{Tr}\left[D_{k}(\mathbf{1}-E \Pi) D_{k-1}(\mathbf{1}-E \Pi) \cdots D_{2}(\mathbf{1}-E \Pi) D_{1} E \Pi\right] .
\end{aligned}
$$

In order to express this product it is convenient to use the notation of the Kronecker product of matrices

$$
\hat{A}=A_{1} \times A_{2}
$$

with the properties

$$
\hat{A} \hat{B}=\left(A_{1} \times A_{2}\right)\left(B_{1} \times B_{2}\right)=A_{1} B_{1} \times A_{2} B_{2}, \quad \operatorname{Tr}(\hat{A})=\operatorname{Tr}\left(A_{1}\right) \operatorname{Tr}\left(A_{2}\right), \quad\left(A_{1} \times A_{2}\right)^{-1}=A_{1}^{-1} \times A_{2}^{-1} .
$$

The second identity, or trace "disentanglement" relation, is relevant for Eq. (15). With the matrix $\hat{C}=(\mathbf{1}-E \Pi) \times(\mathbf{1}-E \Pi)$ it gives us

$$
\left|\phi_{k}\right|^{2}=\operatorname{Tr}\left(\hat{D}_{k} \hat{C} \cdots \hat{D}_{2} \hat{C} \hat{D}_{1} \hat{E} \hat{\Pi}\right)
$$

with $\hat{E}=E \times E, \hat{\Pi}=\Pi \times \Pi$ and $\hat{D}_{k}=D_{k}^{*} \times D_{k}$. For the matrix elements we use the notation

$$
[A \times B]_{i j, k l}=A_{i k} B_{j l} .
$$

\section{AVERAGING OVER THE DISTRIBUTION OF RANDOM TIME STEPS}

In the previous section we obtained a random distribution of return probabilities $\left\{\left|\phi_{k}\right|^{2}\right\}$. Here we are interested in the mean values $\left\{\left\langle\left|\phi_{k}\right|^{2}\right\rangle_{\tau}\right\}$, The subsequent calculation of the time average is based on the 
fact that the random matrices $\left\{\hat{D}_{k}\right\}$ are statistically independent and identically distributed. Thus, from Eq. (17) we get

$$
\left\langle\left|\phi_{k}\right|^{2}\right\rangle_{\tau}=\operatorname{Tr}\left(\left\langle\hat{D}_{k}\right\rangle_{\tau} \hat{C} \cdots\left\langle\hat{D}_{2}\right\rangle_{\tau} \hat{C}\left\langle\hat{D}_{1}\right\rangle_{\tau} \hat{E} \hat{\Pi}\right)=\operatorname{Tr}\left(\left[\langle\hat{D}\rangle_{\tau} \hat{C}\right]^{k-1}\langle\hat{D}\rangle_{\tau} \hat{E} \hat{\Pi}\right)
$$

With the matrices

$$
\hat{\Gamma}=\left\langle D^{*}(\mathbf{1}-E \Pi) \times D(\mathbf{1}-E \Pi)\right\rangle_{\tau}=\left\langle D^{*} \times D\right\rangle_{\tau}(\mathbf{1}-E \Pi) \times(\mathbf{1}-E \Pi)=\langle\hat{D}\rangle_{\tau} \hat{C}
$$

and

$$
\hat{G}=\left\langle D^{*} E \Pi \times D E \Pi\right\rangle_{\tau}=\left\langle D^{*} \times D\right\rangle_{\tau} E \Pi \times E \Pi=\langle\hat{D}\rangle_{\tau} \hat{E} \hat{\Pi}
$$

we obtain the compact expression

$$
\left\langle\left|\phi_{k}\right|^{2}\right\rangle_{\tau}=\operatorname{Tr}\left[\hat{\Gamma}^{k-1} \hat{G}\right] .
$$

$\hat{\Gamma}$ depends on the averaged product $\left\langle D^{*} \times D\right\rangle_{\tau}$. The latter cannot be expressed as a Kronecker product, which prevents us also from applying the trace "disentanglement" relation of Eq. (16). This reflects a robust "entanglement" due to $\left\langle D^{*} \times D\right\rangle_{\tau}$. We will return to this fact in the next section.

\section{A. The generating functions}

First, from Eq. (22) we obtain, after a discrete Fourier transformation, the generating functions

$$
F(\omega)=\sum_{k \geq 1} e^{i k \omega}\left\langle\left|\phi_{k}\right|^{2}\right\rangle_{\tau}=e^{i \omega} \operatorname{Tr}\left[\left(\hat{\mathbf{1}}-e^{i \omega} \hat{\Gamma}\right)^{-1} \hat{G}\right],
$$

and

$$
F_{\tau}(\omega)=\sum_{k \geq 1}\left\langle e^{i \omega\left(\tau_{1}+\cdots+\tau_{k}\right)}\left|\phi_{k}\right|^{2}\right\rangle_{\tau}=\operatorname{Tr}\left[\left(\hat{\mathbf{1}}-\hat{\Gamma}_{\omega}\right)^{-1} \hat{G}_{\omega}\right]
$$

with

$$
\hat{\Gamma}_{\omega}=\left\langle e^{i \omega \tau} \hat{D}\right\rangle_{\tau} \hat{C}
$$

and

$$
\hat{G}_{\omega}=\left\langle e^{i \omega \tau} \hat{D}\right\rangle_{\tau} E \Pi \times E \Pi .
$$

As shown in App. E the matrix $(\hat{\mathbf{1}}-z \hat{\Gamma})^{-1}$ is analytic for $|z|<1$. This means that we should consider the discrete Fourier summation as an analytic continuation $z \rightarrow e^{i \omega}$ from $|z|<1$.

From $F(\omega)$ and $F_{\tau}(\omega)$ we can calculate moments of $k$ and $t_{k}=\tau_{1}+\cdots+\tau_{k}$ with respect to the weight $\left\langle\left|\phi_{k}\right|^{2}\right\rangle_{\tau}$ and $\left\langle\tau\left|\phi_{k}\right|^{2}\right\rangle_{\tau}$, respectively, as

$$
\overline{k^{m}}=\sum_{k \geq 1} k^{m}\left\langle\left|\phi_{k}\right|^{2}\right\rangle_{\tau}=\left.\left(-i \partial_{\omega}\right)^{m} F(\omega)\right|_{\omega=0}, \overline{t^{m}}=\sum_{k \geq 1}\left\langle t_{k}^{m}\left|\phi_{k}\right|^{2}\right\rangle_{\tau}=\left.\left(-i \partial_{\omega}\right)^{m} F_{\tau}(\omega)\right|_{\omega=0} .
$$

The property $F(\omega=0)=F_{\tau}(\omega=0)=1$, discussed in App. A indicates a close relation between the two generating functions. Then the $\omega$ dependence of the generating functions is through the fact that (i) $F(\omega)$ and $F_{\tau}(\omega)$ depend on $\omega$ only through $\left\langle e^{i \omega} \hat{D}\right\rangle_{\tau}$ and $\left\langle e^{i \omega \tau} \hat{D}\right\rangle_{\tau}$, respectively, and (ii) the matrix $\left\langle e^{i \omega \tau} \hat{D}\right\rangle_{\tau}$ is a function of $\omega+E_{j}-E_{j^{\prime}}$. It implies that we can replace a derivative with respect to $\omega$ by a derivative with respect to the difference of energy levels if $E_{j}-E_{j^{\prime}} \neq 0\left(j^{\prime} \neq j\right)$. Since the latter is implicitly assumed here for all energy levels, we can write for the first moment in Eq. (27)

$$
-\left.i \partial_{\omega} F(\omega)\right|_{\omega=0}=\left.\sum_{j, j^{\prime}}\left[\partial_{\bar{D}_{j j^{\prime}}} F(\omega)\right] \partial_{\omega} \bar{D}_{j j^{\prime}}(\omega)\right|_{\omega=0}
$$




$$
=\sum_{j \neq j^{\prime}}\left[\partial_{\bar{D}_{j j^{\prime}}(0)} F(0)\right] \partial_{E_{j}-E_{j^{\prime}}} \bar{D}_{j j^{\prime}}(0)+\left.\sum_{j}\left[\partial_{\bar{D}_{j j}(\omega)} F(\omega)\right] \partial_{\omega} \bar{D}_{j j}(\omega)\right|_{\omega=0}
$$

with $\bar{D}_{j j^{\prime}}(\omega)=e^{i \omega}\left\langle\hat{D}_{j j^{\prime}}\right\rangle_{\tau}$. The first sum on the right-hand side vanishes due to $F(0)=1$ and consequently $\partial_{E_{j}-E_{j^{\prime}}} F(0)=0$, such that we obtain

$$
\left.\partial_{\omega} F(\omega)\right|_{\omega=0}=\left.\sum_{j} \partial_{\bar{D}_{j j}(\omega)} F(\omega)\right|_{\omega=0} i\left\langle\hat{D}_{j j}(0)\right\rangle_{\tau}=\left.i \sum_{j} \partial_{\bar{D}_{j j}(\omega)} F(\omega)\right|_{\omega=0} .
$$

The analog calculation is valid for $F_{\tau}(\omega)$ and gives

$$
\left.\partial_{\omega} F_{\tau}(\omega)\right|_{\omega=0}=\left.\sum_{j} \partial_{\bar{D}_{j j}(\omega)} F_{\tau}(\omega)\right|_{\omega=0} i\left\langle\tau \hat{D}_{j j}(0)\right\rangle_{\tau}=\left.i\langle\tau\rangle_{\tau} \sum_{j} \partial_{\bar{D}_{j j}(\omega)} F(\omega)\right|_{\omega=0} .
$$

Comparing the expressions in Eqs. (28) and (29) implies for the first moments in Eq. (27) the relation

$$
\bar{t}=\sum_{k \geq 1}\left\langle t_{k}\left|\phi_{k}\right|^{2}\right\rangle_{\tau}=\langle\tau\rangle_{\tau} \sum_{k \geq 1} k\left\langle\left|\phi_{k}\right|^{2}\right\rangle_{\tau}=\langle\tau\rangle_{\tau} \bar{k}
$$

\section{B. Evaluation of $\left\langle|\tilde{\phi}(\omega)|^{2}\right\rangle_{\tau}$}

Next we will show that $\left\langle|\tilde{\phi}(\omega)|^{2}\right\rangle_{\tau}=1$ holds in general for any integer $N$ due to

$$
T_{j_{1} j_{2}}=\sum_{j_{3}, j_{4}}\left[\langle\hat{D}\rangle_{\tau}^{-1}-\hat{C}\right]_{j_{1} j_{2}, j_{3} j_{4}}^{-1}=\frac{1}{p_{j_{1}}} \delta_{j_{1} j_{2}}
$$

where $\hat{C}$ was defined in Sect. $\mathrm{V}$ To derive this property and to calculate the mean FDR time we use the matrix relations

$$
\hat{E} \hat{W}\left[\langle\hat{D}\rangle_{\tau}^{-1}-\hat{C}\right]=\hat{E} \hat{\Pi}, \quad\left[\langle\hat{D}\rangle_{\tau}^{-1}-\hat{C}\right] \hat{T} \hat{E}=\hat{E},
$$

where $\hat{W}$ is the $N^{2} \times N^{2}$ diagonal matrix $\operatorname{diag}\left(p_{1}, 0_{N}, p_{2}, 0_{N}, \ldots, 0_{N}, p_{N}\right)$, and $0_{N}$ is a sequence of $N$ zeros. $\hat{T}$ is the $N^{2} \times N^{2}$ diagonal matrix with elements $T_{j j^{\prime}}=\delta_{j j^{\prime}} / p_{j}$ of Eq. (31). The $\langle\hat{D}\rangle_{\tau}$ contribution on the right-hand side disappears, since $\hat{W}\langle\hat{D}\rangle_{\tau}=\hat{W}$. The first relation of Eq. (32) is obtained from $W_{j_{1} j_{2}}=p_{j_{1}} \delta_{j_{1} j_{2}}$ by a direct inspection of the matrix elements:

$$
\sum_{j_{1}, j_{2}} W_{j_{1} j_{2}}\left[-E_{j_{1} j_{3}} p_{j_{3}} E_{j_{2} j_{4}} p_{j_{4}}+E_{j_{1} j_{3}} p_{j_{3}} \delta_{j_{2} j_{4}}+\delta_{j_{1} j_{3}} E_{j_{2} j_{4}} p_{j_{4}}\right]=p_{j_{3}} p_{j_{4}}
$$

and the second relation for $T_{j_{3} j_{4}}=\delta_{j_{3} j_{4}} / p_{j_{3}}$ from

$$
\sum_{j_{3}, j_{4}}\left[-E_{j_{1} j_{3}} p_{j_{3}} E_{j_{2} j_{4}} p_{j_{4}}+E_{j_{1} j_{3}} p_{j_{3}} \delta_{j_{2} j_{4}}+\delta_{j_{1} j_{3}} E_{j_{2} j_{4}} p_{j_{4}}\right] T_{j_{3} j_{4}}=1
$$

Provided that the inverse of $\langle\hat{D}\rangle_{\tau}^{-1}-\hat{C}$ exists, the second relation of Eq. (32) implies $\left[\langle\hat{D}\rangle_{\tau}^{-1}-\hat{C}\right]^{-1} \hat{E}=$ $\hat{T} \hat{E}$, which gives directly Eq. (31) and subsequently the normalization

$$
\left\langle|\tilde{\phi}(\omega)|^{2}\right\rangle_{\tau}=1
$$

according to Eq. (D8) of App. D This result can be used to reduce the integral of the average winding number

$$
\langle w\rangle_{\tau}:=\frac{1}{2 \pi} \int_{0}^{2 \pi} \frac{\left\langle\tilde{\phi}(\omega)^{*}\left[-i \partial_{\omega}\right] \tilde{\phi}(\omega)\right\rangle_{\tau}}{\left\langle|\tilde{\phi}(\omega)|^{2}\right\rangle_{\tau}} d \omega,
$$

which is discussed in more detail in Sect. VIII, with the help of Eq. (33) to

$$
\langle w\rangle_{\tau}=\frac{1}{2 \pi} \int_{0}^{2 \pi}\left\langle\tilde{\phi}(\omega)^{*}\left[-i \partial_{\omega}\right] \tilde{\phi}(\omega)\right\rangle_{\tau} d \omega=\sum_{k \geq 1} k\left\langle\left|\phi_{k}\right|^{2}\right\rangle_{\tau}=\bar{k} .
$$

In the next section we will see that $\langle w\rangle_{\tau}=\bar{k}$ is an integer, equal to the dimensionality of the accessible Hilbert space. 


\section{Mean FDR time}

Now we return to the first moment in Eq. (27), using an extension of the previous calculation. Starting with

$$
-\left.i \partial_{\omega} F(\omega)\right|_{\omega=0}=\operatorname{Tr}\left[(\hat{\mathbf{1}}-\hat{\Gamma})^{-2}\langle\hat{D}\rangle_{\tau} \hat{E} \hat{\Pi}\right]
$$

we write for the matrix inside the trace

$$
\hat{E} \hat{\Pi}\left[\langle\hat{D}\rangle_{\tau}^{-1}-\hat{C}\right]^{-1}\langle\hat{D}\rangle_{\tau}^{-1}\left[\langle\hat{D}\rangle_{\tau}^{-1}-\hat{C}\right]^{-1}
$$

and apply the first relation of Eq. (32) to the first inverse matrix to obtain

$$
=\hat{E} \hat{W}\langle\hat{D}\rangle_{\tau}^{-1}\left[\langle\hat{D}\rangle_{\tau}^{-1}-\hat{C}\right]^{-1}=\hat{E} \hat{W}\left[\langle\hat{D}\rangle_{\tau}^{-1}-\hat{C}\right]^{-1},
$$

where the last equation is due to $\hat{W}\langle\hat{D}\rangle_{\tau}^{-1}=\hat{W}$. This can be inserted into Eq. (36), and with the second relation of Eq. (32) we get

$$
-\left.i \partial_{\omega} F(\omega)\right|_{\omega=0}=\operatorname{Tr}\left\{\hat{E} \hat{W}\left[\langle\hat{D}\rangle_{\tau}^{-1}-\hat{C}\right]^{-1}\right\}=\operatorname{Tr}\{\hat{W} \hat{T} \hat{E}\}=\sum_{j_{1}=1}^{N} T_{j_{1} j_{1}} p_{j_{1}}=N,
$$

where the last two equations follow from Eq. (31) and the definition of $\hat{W}$. This result gives us, together with Eqs. (30) and (38), for the mean number of measurements (MNM) of the FDR and the mean FDR time

$$
\bar{k}=\sum_{k \geq 1} k\left\langle\left|\phi_{k}\right|^{2}\right\rangle_{\tau}=\langle w\rangle_{\tau}=N \text { and } \bar{t}=\sum_{k \geq 1}\left\langle t_{k}\left|\phi_{k}\right|^{2}\right\rangle_{\tau}=\langle\tau\rangle_{\tau}\langle w\rangle_{\tau}=\langle\tau\rangle_{\tau} N
$$

which presents an extension of a central result of the seminal work by Grünbaum et al. [9] of stroboscopic measurements to random time measurements.

As already mentioned in the Introduction, higher order moments are not quantized but can be very sensitive to degeneracies of the spectrum, at least for stroboscopic measurements [3, 11, 18]. In the case of random measurements this is also true near degeneracies of the energy levels when $\left\langle D_{j}^{*} D_{j^{\prime}}\right\rangle_{\tau}=$ $\left\langle e^{i\left(E_{j}-E_{j^{\prime}}\right) \tau}\right\rangle_{\tau}\left(j^{\prime} \neq j\right)$ is close to 1 . This originates in the fact that for

$$
-\left.\partial_{\omega}^{2} F(\omega)\right|_{\omega=0}=\operatorname{Tr}\left[(\hat{\mathbf{1}}-\hat{\Gamma})^{-3}\langle\hat{D}\rangle_{\tau} \hat{E} \hat{\Pi}\right]=\operatorname{Tr}\left\{\hat{W}(\hat{\mathbf{1}}-\hat{\Gamma})^{-1} \hat{T} \hat{E}\right\}
$$

small eigenvalues of $\hat{\mathbf{1}}-\hat{\Gamma}$ can appear. This can indeed happen when at least one $\left\langle D_{j}^{*} D_{j^{\prime}}\right\rangle_{\tau}$ is close to 1 or when one $p_{j}$ is close to 0 , as shown in App. E

\section{EXAMPLE: SYMMETRIC TWO-LEVEL SYSTEM}

In the previous section we derived relations between the MNM of the FDR and the mean FDR time, their relation with the average winding number $\langle w\rangle_{\tau}$ of Eq. (34) and with the dimensionality of the Hilbert space in Eq. (39). These results are general and valid for any quantum system on an $N$-dimensional Hilbert space. Besides these mean values we obtained in Eq. (27) also higher moments of the number of measurements and the return time for the FDR. For $m>1$ we have not found a simple expression but can obtain these moments only by calculating the generating functions $F(\omega)$ and $F_{\tau}(\omega)$ directly. To determine these generating functions would require the inversion of the $N^{2} \times N^{2}$ matrix $\hat{\mathbf{1}}-z \hat{\Gamma}$. This is a tedious task, which goes beyond the scope of this paper. Therefore, we limit ourselves to $N=2$ and calculate the corresponding $4 \times 4$ matrices (cf. App. F). In particular, we consider a symmetric two-level system (2LS) with energy levels $E_{ \pm}= \pm J$ and spectral weights $p_{1}=p_{2}=1 / 2$ for random times. The Hilbert space is two-dimensional with two basis states, e.g. $|0\rangle$ and $|1\rangle$. If the measured state is $|0\rangle$, the 
projector $P$ reads $P=|1\rangle\langle 1|$. Then we get $\left\langle 0\left|e^{-i H \tau}\right| 0\right\rangle=\cos (J \tau)$ and $\left\langle 0\left|e^{-i H \tau}\right| 1\right\rangle=i \sin (J \tau)$ and the return amplitude $\phi_{k}$ reads

$$
\phi_{k}=\left\{\begin{array}{ll}
\cos \left(J \tau_{1}\right) & \text { for } k=1 \\
-\sin \left(J \tau_{1}\right) \sin \left(J \tau_{2}\right) & \text { for } k=2 \\
-\sin \left(J \tau_{1}\right) \cos \left(J \tau_{2}\right) \cdots \cos \left(J \tau_{k-1}\right) \sin \left(J \tau_{k}\right) & \text { for } k \geq 3
\end{array} .\right.
$$

The simplicity of the two-level system is manifested in the fact that $\phi_{k}$ is a scalar product in contrast to the matrix product in Eq. (14) of the general case $N>2$. This simplifies calculations with respect to random $\left\{\tau_{k}\right\}$ substantially. For instance, we can easily perform the summation with respect to $k$ to get

$$
\sum_{k=1}^{n}\left|\phi_{k}\right|^{2}=1-\left[1-\cos ^{2}\left(J \tau_{1}\right)\right] \cos ^{2}\left(J \tau_{2}\right) \cdots \cos ^{2}\left(J \tau_{n}\right)
$$

as a special case of the general equation A1 . After averaging with respect to $\left\{\tau_{k}\right\}$, we get for the mean FDR time

$$
\bar{t}=\sum_{k \geq 1}\left\langle\left(\tau_{1}+\cdots+\tau_{k}\right)\left|\phi_{k}\right|^{2}\right\rangle_{\tau}=2\langle\tau\rangle_{\tau}
$$

which is in agreement with our general result in Eq. (39). Moreover, the generating functions $F(\omega)$ in Eq. (23) and $F_{\tau}(\omega)$ in Eq. (24) read

$$
F(\omega)=e^{i \omega} \frac{\left(2 e^{i \omega}-1\right)\langle\cos 2 J \tau\rangle_{\tau}-1}{e^{i \omega}\left(\langle\cos 2 J \tau\rangle_{\tau}+1\right)-2}, \quad F_{\tau}(\omega)=\frac{\left(2\left\langle e^{i \omega \tau}\right\rangle_{\tau}-1\right)\left\langle e^{i \omega \tau} \cos 2 J \tau\right\rangle_{\tau}-\left\langle e^{i \omega \tau}\right\rangle_{\tau}}{\left\langle e^{i \omega \tau} \cos 2 J \tau\right\rangle_{\tau}+\left\langle e^{i \omega \tau}\right\rangle_{\tau}-2},
$$

which gives for $\omega=0$

$$
\sum_{k \geq 1}\left\langle\left|\phi_{k}\right|^{2}\right\rangle_{\tau}=F(0)=1, \quad \sum_{k \geq 1} k\left\langle\left|\phi_{k}\right|^{2}\right\rangle_{\tau}=-i F^{\prime}(0)=2, \quad \sum_{k \geq 1} k^{2}\left\langle\left|\phi_{k}\right|^{2}\right\rangle_{\tau}=-F^{\prime \prime}(0)=2 \frac{3-\langle\cos 2 J \tau\rangle_{\tau}}{1-\langle\cos 2 J \tau\rangle_{\tau}}
$$

where the first equation reflects the normalization, the second equation the quantization of the mean FDR time and the last equation the FDR fluctuations. The latter only diverge for $J=0$ in the case of random times steps, but in the case of a fixed time step $\tau$ it also diverges for $J \tau=k \pi(k= \pm 1, \pm 2, \ldots)$. For $F_{\tau}(\omega)$ we get

$$
\sum_{k \geq 1}\left\langle\left(\tau_{1}+\cdots+\tau_{k}\right)\left|\phi_{k}\right|^{2}\right\rangle_{\tau}=-i F_{\tau}^{\prime}(0)=2\langle\tau\rangle_{\tau}
$$

The two-level systems gives also a direct insight into the effect of random time steps on the return amplitude $\tilde{\phi}(\omega)$ before averaging, since we can calculate these amplitudes from Eq. (40) for special realizations of $\left\{\tau_{k}\right\}$. A few examples are visualized in Fig. 1, indicating that $|\tilde{\phi}(\omega)|$ as well as the winding number vary from realization to realization substantially. For fixed time steps, on the other hand, we have $|\tilde{\phi}(\omega)|=1$ and the winding number is 2 in Fig. 2. as predicted by the general theory of stroboscopic measurements [9, 18].

\section{DISCUSSION}

The aim of our work has been to calculate the properties of the ME with random measurements through the return amplitude $\tilde{\phi}(\omega)$. For given eigenvalues $\left\{E_{j}\right\}$ and eigenstates $\left\{\left|E_{j}\right\rangle\right\}$ of the Hamiltonian $H$ the ME of the return to the initial state $|\Psi\rangle$ are characterized by the time averaged phase factors $\left\langle e^{ \pm i E_{j} \tau}\right\rangle_{\tau}$, $\left\langle e^{i\left(E_{j}-E_{j}^{\prime}\right) \tau}\right\rangle_{\tau}$ and by the spectral weights $p_{j}=\left|\left\langle E_{j} \mid \Psi\right\rangle\right|^{2}$. This allowed us to calculated the mean FDR times and the MNM of the FDR, using the generating functions defined in Eqs. (23) and (24). The surprising result in Eq. (39) is that the MNM of the FDR is just the dimensionality $N$ of the accessible Hilbert space and that the mean FDR time is $\langle\tau\rangle_{\tau} N$, where $\langle\tau\rangle_{\tau}$ is the mean time between two successive measurements. The same was previously observed for fixed time steps $\tau[9,11$, 18]. The robustness of the 

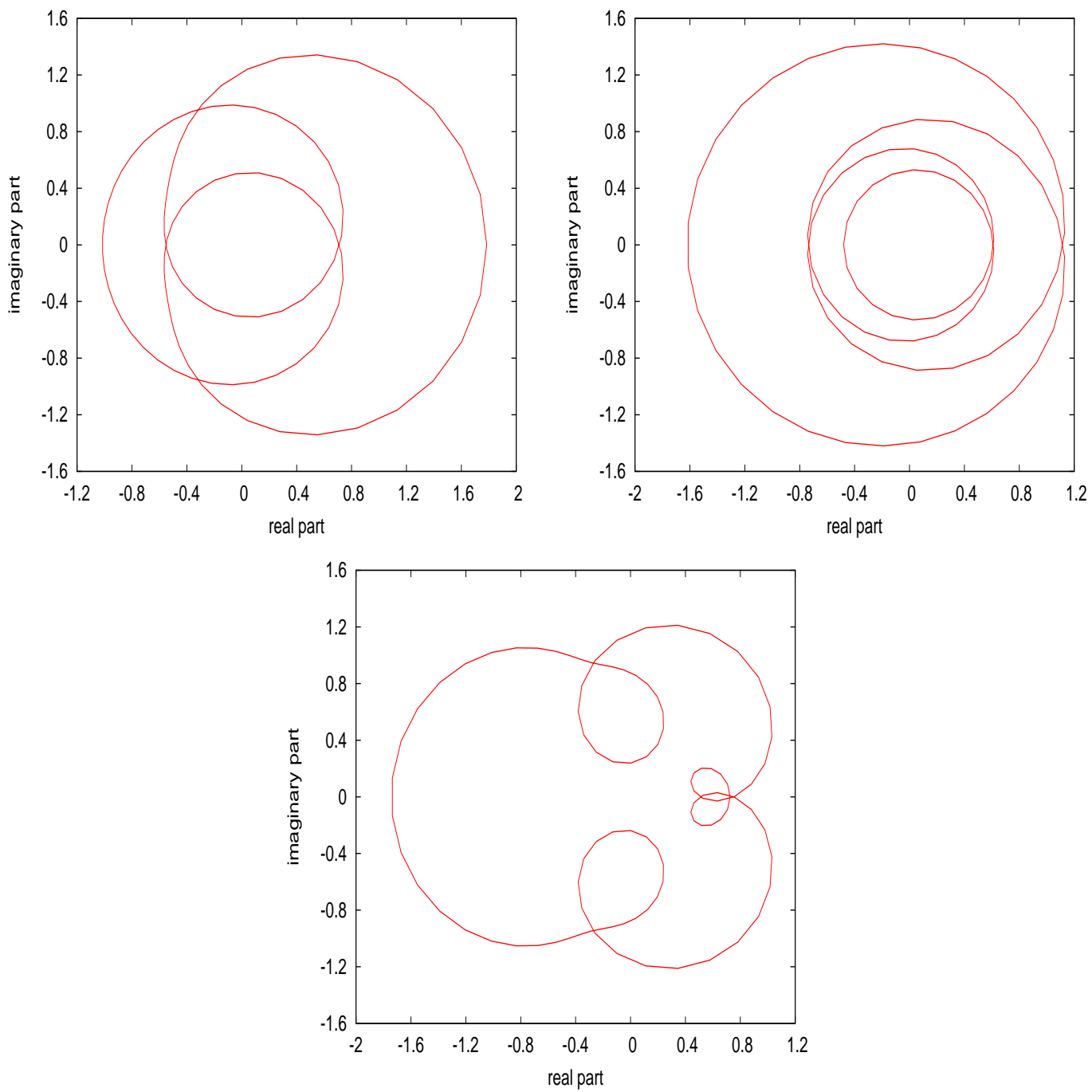

FIG. 1. Symmetric two-level system: The return amplitude $\tilde{\phi}_{8}(\omega)=\sum_{k=1}^{8} e^{i k \omega} \phi_{k}$ on the $\omega$ interval $[0,2 \pi)$ with three randomly chosen realizations of $\left\{\tau_{1}, \ldots, \tau_{8}\right\}$ performs a closed trajectory in the complex plane. The winding numbers in these examples are $w_{\phi}=3,4,1$, respectively.

average winding number is remarkable, since the winding number of the return amplitude $\tilde{\phi}(\omega)$ fluctuates strongly from realization to realization of the random $\left\{\tau_{k}\right\}$ (cf. Fig. 11).

Other quantities, such as the correlation function $\left\langle\phi_{k}^{*} \phi_{k^{\prime}}\right\rangle_{\tau}$ of the return amplitudes for different discrete times $k$ and $k^{\prime}$ in Eq. (C1), can also be calculated. An example is $\sum_{k, k^{\prime}>1} e^{i\left(k^{\prime}-k\right) \omega}\left\langle\phi_{k}^{*} \phi_{k^{\prime}}\right\rangle_{\tau}=\left\langle|\tilde{\phi}(\omega)|^{2}\right\rangle_{\tau}$, which is 1 according to the calculation in App. D. This enabled us to determine the mean FDR time in Eq. (39). Its connection with the integral in Eq. (34) is a generalization of the quantized winding number $w_{s t}$ in the case of stroboscopic measurements by Grünbaum et al. [9, 18]. The latter is based on the fact that $|\tilde{\phi}(\omega)|=1$ (cf. Eq.(12)). Then the winding number $w_{s t}$ simply reads for $\tilde{\phi}(\omega)=e^{i \varphi}$ (cf. Ref. [3] and Sect. [IV]

$$
w_{s t}=-\frac{i}{2 \pi} \int_{0}^{2 \pi} \tilde{\phi}(\omega)^{*} \partial_{\omega} \tilde{\phi}(\omega) d \omega=\frac{1}{2 \pi} \int_{0}^{2 \pi} \partial_{\omega} \varphi d \omega
$$

In the case of random time measurements $\left|\tilde{\phi}\left(\omega ;\left\{\tau_{k}\right\}\right)\right| \neq 1$, such that we must modify the definition of the winding number by normalizing $\tilde{\phi}\left(\omega,\left\{\tau_{k}\right\}\right)$. A further problem is that the winding number depends 


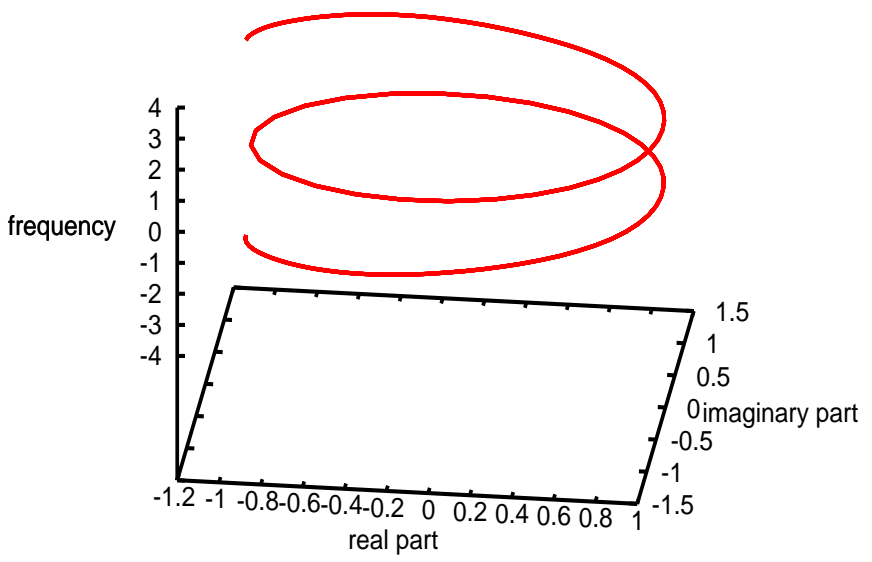

FIG. 2. Symmetric two-level system: The return amplitude $\tilde{\phi}_{8}(\omega)=\sum_{k=1}^{8} e^{i k \omega} \phi_{k}$ on the $\omega$ interval $[0,2 \pi)$ with fixed time step $\tau \approx \pi / 2 J$. The winding number in this example is $w_{\phi}=2$.

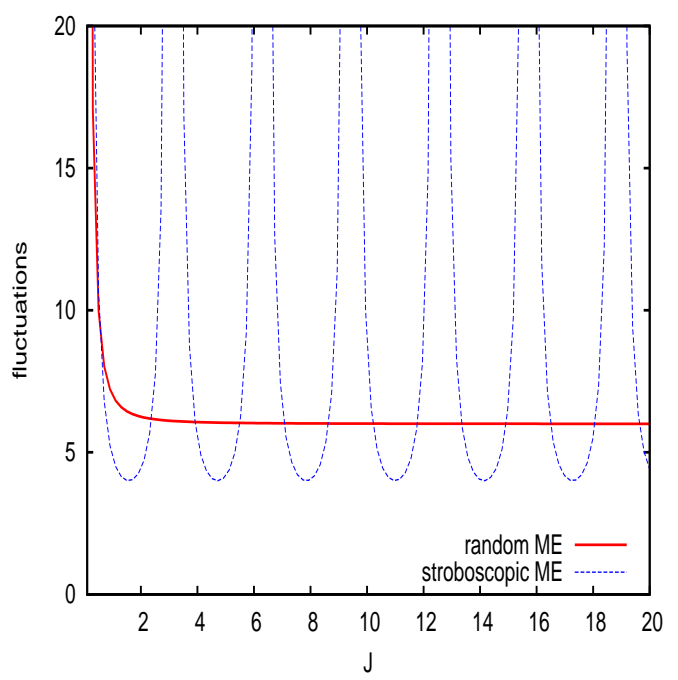

FIG. 3. Symmetric two-level system: Fluctuations of the FDR for stroboscopic ME and random time steps from Eqs. (48) and (49).

on the realization of the time steps $\left\{\tau_{k}\right\}$, as we have demonstrated in Fig. 1 for the symmetric two-level system. Therefore, we must also perform an average with respect to the time steps. Then the definition of the winding number becomes Eq. (34), which reads with $\tilde{\phi}(\omega)=|\tilde{\phi}(\omega)| e^{i \varphi}$ as an average differential phase change

$$
\langle w\rangle_{\tau}=\frac{1}{2 \pi} \int_{0}^{2 \pi} \frac{\left\langle|\tilde{\phi}(\omega)|^{2} \partial_{\omega} \varphi\right\rangle_{\tau}}{\left\langle|\tilde{\phi}(\omega)|^{2}\right\rangle_{\tau}} d \omega
$$

Here we note that Eq. (34) is formally equivalent to the definition of the Berry phase [27] when we replace $\tilde{\phi}\left(\omega,\left\{\tau_{k}\right\}\right)$ by the spatial wave function $\phi(\omega, \mathbf{r})$ and replace the time average $\langle\ldots\rangle_{\tau}$ by the usual quantum average in space.

Averaging over random time steps is crucial to obtain a generic winding number. That different special 
realizations of the random time steps lead to different winding numbers can be seen when we assume a finite sequence of $\left\{\phi_{k}\right\}(k \leq M)$. The latter is either the result of an approximative truncation of the sequence or when the sequence terminates with $S_{M}=0$ in Eq. (A1). Then the Fourier transformed return amplitude in Eq. (5) becomes a finite sum

$$
\tilde{\phi}_{M}(\omega)=\sum_{k=1}^{M} e^{i \omega k} \phi_{k}
$$

for which a winding number $w_{M}$ can be defined for (random) coefficients $\left\{\phi_{k}\right\}$ as

$$
w_{M}=\frac{1}{2 \pi} \int_{0}^{2 \pi} \frac{\tilde{\phi}_{M}^{*}(\omega)\left(-i \partial_{\omega}\right) \tilde{\phi}_{M}(\omega)}{\tilde{\phi}_{M}^{*}(\omega) \tilde{\phi}_{M}(\omega)} d \omega=\frac{1}{2 \pi} \int_{0}^{2 \pi}\left(-i \partial_{\omega}\right) \log \left[\tilde{\phi}_{M}(\omega)\right] d \omega .
$$

Then we rewrite the polynomial $\tilde{\phi}_{M}(\omega)$ as the product

$$
\tilde{\phi}_{M}(\omega)=\phi_{M}\left(e^{i \omega}-z_{1}\right) \cdots\left(e^{i \omega}-z_{M}\right),
$$

such that we get for the winding number

$$
w_{M}=\sum_{k=1}^{M} \frac{1}{2 \pi} \int_{0}^{2 \pi} \frac{e^{i \omega}}{e^{i \omega}-z_{k}} d \omega .
$$

With $z=e^{i \omega}$ this gives

$$
w_{M}=\sum_{k=1}^{M} \frac{1}{2 \pi i} \int_{S_{1}} \frac{1}{z-z_{k}} d z=M^{\prime},
$$

where the Cauchy integral is performed over the unit circle $S_{1}$ and $M^{\prime}\left(0 \leq M^{\prime} \leq M\right)$ is the number of poles inside the unit circle. A simple example is $M=2$ with

$$
\tilde{\phi}_{2}(\omega)=\phi_{1} e^{i \omega}+\phi_{2} e^{2 i \omega}=z\left(\phi_{1}+\phi_{2} z\right)=\phi_{2} z\left(z+\phi_{1} / \phi_{2}\right),
$$

where we get

$$
w_{2}=\left\{\begin{array}{ll}
1 & \left|\phi_{1} / \phi_{2}\right|>1 \\
2 & \left|\phi_{1} / \phi_{2}\right|<1
\end{array} .\right.
$$

A detailed calculation of several quantities was presented in Sect. VII for the case of a symmetric twolevel system with $w=2$ after averaging with respect to $\left\{\tau_{k}\right\}$. The fluctuations of the return time are finite

$$
\sum_{k \geq 1} k^{2}\left\langle\left|\phi_{k}\right|^{2}\right\rangle_{\tau}=2 \frac{3-\langle\cos 2 J \tau\rangle_{\tau}}{1-\langle\cos 2 J \tau\rangle_{\tau}}=\frac{1+6 J^{2}}{J^{2}},
$$

where the last expression is obtained from the Poisson distribution $e^{-\tau_{k}} d \tau_{k}$. In the limit of a fixed measurement time $\tau$ the fluctuations

$$
\sum_{k \geq 1} k^{2}\left|\phi_{k}\right|^{2}=2 \frac{3-\cos 2 J \tau}{1-\cos 2 J \tau}
$$

would diverge for $J \tau=\pi n(n=0,1, \ldots)$. Thus, the random measurements wash out the divergences of the fluctuations. For most values of the level splitting $J$ the fluctuations are stronger for the fixed time steps, as visualized in Fig. 3.

In this paper we have completely focused on the return of the quantum system to its initial state. A natural extension would be a corresponding analysis of the transition from an initial to a different final state, monitored by random projective measurements. We have addressed this topic in a separate article [?].

In conclusion, the mean FDR time of the ME for random time steps is equal to the dimensionality of the accessible Hilbert space. This is very similar to the ME for fixed time steps. On the other hand, the strong fluctuations of the FDR time, which appear for a small distance of eigenvalues in the case of fixed time steps, are washed out by averaging with respect to the random time steps. This was briefly discussed for a two-level system in this article and more general in Ref. [? ]. 


\begin{tabular}{|l|l|l|}
\hline & SME & RME \\
\hline probability $|\tilde{\phi}(\omega)|^{2}$ & 1 & random \\
probability $\left\langle|\tilde{\phi}(\omega)|^{2}\right\rangle_{\tau} \tilde{l}$ & - & 1 \\
winding number $w$ of $\tilde{\phi}(\omega)$ & $N$ & random \\
winding number $\langle w\rangle_{\tau}$ of $\tilde{\phi}(\omega)$ & - & $N$ \\
mean number of measurements for FDR $\bar{k}$ & $N$ & $N$ \\
mean FDR time $\bar{t}$ & $N \tau$ & $N\langle\tau\rangle_{\tau}$ \\
\hline
\end{tabular}

TABLE I. Comparison between stroboscopic ME (SME) and random ME (RME) with the Hilbert space dimensionality $N$. The first detected return agrees for both approaches, provided that we average over the random measurements.

\section{ACKNOWLEDGMENTS:}

The support of Israel Science Foundation's Grant No. 1898/17 as well as the support by the Julian Schwinger Foundation (K.Z.) are acknowledged.

\section{Appendix A: Normalization}

The normalization of the vector $\vec{\phi}=\left(\phi_{1}, \phi_{2}, \ldots, \phi_{n}\right)$ with

$$
\phi_{k}=\left\langle\Psi\left|e^{-i H \tau_{k}}\left(P e^{-i H \tau_{k-1}}\right) \cdots\left(P e^{-i H \tau_{1}}\right)\right| \Psi\right\rangle
$$

in the limit $n \rightarrow \infty$ is based on the normalization of $|\Psi\rangle$

$$
\left\langle\Psi\left|e^{i H \tau_{1}} e^{-i H \tau_{1}}\right| \Psi\right\rangle=\langle\Psi \mid \Psi\rangle=1
$$

and will be derived by iteration: For the evaluation of $|\vec{\phi}|^{2}$ we consider the sequence of projection operators $\left\{\Pi_{k}\right\}_{k=1,2, \ldots, n}$ with

$$
\Pi_{k}:=P e^{i H \tau_{k}} e^{-i H \tau_{k}} P, \quad P=\mathbf{1}-|\Psi\rangle\langle\Psi| \equiv \mathbf{1}-P_{0} .
$$

With $\Pi_{k}=P^{2}=P$ and $|\Psi\rangle\langle\Psi|+P=\mathbf{1}$ we can insert $P_{0}+\Pi_{2}=\mathbf{1}$ at

$$
\begin{aligned}
& 1=\left\langle\Psi\left|e^{i H \tau_{1}} e^{-i H \tau_{1}}\right| \Psi\right\rangle=\left\langle\Psi\left|e^{i H \tau_{1}}\left(P_{0}+\Pi_{2}\right) e^{-i H \tau_{1}}\right| \Psi\right\rangle \\
& =\left\langle\Psi\left|e^{i H \tau_{1}}\right| \Psi\right\rangle\left\langle\Psi\left|e^{-i H \tau_{1}}\right| \Psi\right\rangle+\left\langle\Psi\left|e^{i H \tau_{1}} \Pi_{2} e^{-i H \tau_{1}}\right| \Psi\right\rangle .
\end{aligned}
$$

Next we replace $\Pi_{2}$ in the second term by

$$
\Pi_{2}=P e^{i H \tau_{2}} e^{-i H \tau_{2}} P=P e^{i H \tau_{2}}\left(P_{0}+\Pi_{3}\right) e^{-i H \tau_{2}} P
$$

to get

$$
\begin{gathered}
1=\left\langle\Psi\left|e^{i H \tau_{1}}\right| \Psi\right\rangle\left\langle\Psi\left|e^{-i H \tau_{1}}\right| \Psi\right\rangle+\left\langle\Psi\left|e^{i H \tau_{1}} P e^{i H \tau_{2}}\left(P_{0}+\Pi_{3}\right) e^{-i H \tau_{2}} P e^{-i H \tau_{1}}\right| \Psi\right\rangle \\
=\left\langle\Psi\left|e^{i H \tau_{1}}\right| \Psi\right\rangle\left\langle\Psi\left|e^{-i H \tau_{1}}\right| \Psi\right\rangle+\left\langle\Psi\left|e^{i H \tau_{1}} P e^{i H \tau_{2}}\right| \Psi\right\rangle\left\langle\Psi\left|e^{-i H \tau_{2}} P e^{-i H \tau_{1}}\right| \Psi\right\rangle \\
+\left\langle\Psi\left|e^{i H \tau_{1}} P e^{i H \tau_{2}} \Pi_{3} e^{-i H \tau_{2}} P e^{-i H \tau_{1}}\right| \Psi\right\rangle
\end{gathered}
$$

The replacement of the operator $\Pi_{k}$ by $P e^{i H \tau_{k}}\left(P_{0}+\Pi_{k+1}\right) e^{-i H \tau_{k}} P$ can be repeated for $k=3, \ldots, n$ to obtain

$$
1=\left\langle\Psi\left|e^{i H \tau_{1}}\right| \Psi\right\rangle\left\langle\Psi\left|e^{-i H \tau_{1}}\right| \Psi\right\rangle+\left\langle\Psi\left|e^{i H \tau_{1}} P e^{i H \tau_{2}}\right| \Psi\right\rangle\left\langle\Psi\left|e^{-i H \tau_{2}} P e^{-i H \tau_{1}}\right| \Psi\right\rangle
$$




$$
\begin{gathered}
+\cdots+\left\langle\Psi\left|e^{i H \tau_{1}}\left(P e^{i H \tau_{2}}\right) \cdots\left(P e^{i H \tau_{n}}\right)\right| \Psi\right\rangle\left\langle\Psi\left|\left(e^{-i H \tau_{n}} P\right) \cdots\left(e^{-i H \tau_{2}} P\right) e^{-i H \tau_{1}}\right| \Psi\right\rangle \\
+\left\langle\Psi\left|e^{i H \tau_{1}}\left(P e^{i H \tau_{2}}\right) \cdots\left(P e^{i H \tau_{n}}\right) P\left(e^{-i H \tau_{n}} P\right) \cdots\left(e^{-i H \tau_{2}} P\right) e^{-i H \tau_{1}}\right| \Psi\right\rangle \\
=\sum_{k=1}^{n}\left|\phi_{k}\right|^{2}+S_{n},
\end{gathered}
$$

where

$$
S_{n}=\left\langle\Psi\left|e^{i H \tau_{1}}\left(P e^{i H \tau_{2}}\right) \cdots\left(P e^{i H \tau_{n}}\right) P\left(e^{-i H \tau_{n}} P\right) \cdots\left(e^{-i H \tau_{2}} P\right) e^{-i H \tau_{1}}\right| \Psi\right\rangle .
$$

Thus, we have

$$
\sum_{k=1}^{n}\left|\phi_{k}\right|^{2}=1-S_{n}
$$

where the probability $S_{n}$ of not recording the state after $n$ attempts is

$$
S_{n}=\left\langle\Psi\left|e^{i H \tau_{1}}\left(P e^{i H \tau_{2}}\right) \cdots\left(P e^{i H \tau_{n}}\right) P\left(e^{-i H \tau_{n}} P\right) \cdots\left(e^{-i H \tau_{2}} P\right) e^{-i H \tau_{1}}\right| \Psi\right\rangle .
$$

Provided the remainder $S_{n}$ vanishes in the limit $n \rightarrow \infty$, the wave function $\vec{\phi}=\left(\phi_{1}, \phi_{2}, \ldots, \phi_{n}\right)$ is normalized in this limit: $|\vec{\phi}|^{2}=1$. Although we do not have proof that $S_{n}$ always vanishes with $n \rightarrow \infty$, the latter is plausible and is supported by the example of the symmetric two-level system in Eq. (41), by stroboscopic measurements [9] and in the case of the time averaged sum $\sum_{k \geq 1}\left\langle\left|\phi_{k}\right|^{2}\right\rangle_{\tau}=1$ [? ].

\section{Appendix B: Recursion}

For an operator $K$ and the projector $|\Psi\rangle\langle\Psi|$ we assume that the inverse $(K+|\Psi\rangle\langle\Psi|)^{-1}$ exists. Then we get the relation

$$
\left\langle\Psi\left|(K+|\Psi\rangle\langle\Psi|)^{-1}\right| \Psi_{0}\right\rangle=\left\langle\Psi\left|K^{-1}\left(\mathbf{1}+|\Psi\rangle\langle\Psi| K^{-1}\right)^{-1}\right| \Psi_{0}\right\rangle .
$$

Now we can use the identity

$$
\left(\mathbf{1}+|\Psi\rangle\langle\Psi| K^{-1}\right)^{-1}=\mathbf{1}-|\Psi\rangle\langle\Psi| K^{-1}\left(\mathbf{1}+|\Psi\rangle\langle\Psi| K^{-1}\right)^{-1}
$$

to write for the right-hand side of Eq. (B1)

$$
\left\langle\Psi\left|K^{-1}\right| \Psi_{0}\right\rangle-\left\langle\Psi\left|K^{-1}\right| \Psi\right\rangle\left\langle\Psi\left|K^{-1}\left(\mathbf{1}+|\Psi\rangle\langle\Psi| K^{-1}\right)^{-1}\right| \Psi_{0}\right\rangle,
$$

such that Eq. (B1) becomes

$$
\left\langle\Psi\left|(K+|\Psi\rangle\langle\Psi|)^{-1}\right| \Psi_{0}\right\rangle=\left\langle\Psi\left|K^{-1}\right| \Psi_{0}\right\rangle-\left\langle\Psi\left|K^{-1}\right| \Psi\right\rangle\left\langle\Psi\left|(K+|\Psi\rangle\langle\Psi|)^{-1}\right| \Psi_{0}\right\rangle .
$$

This relation is equivalent to

$$
\left\langle\Psi\left|(K+|\Psi\rangle\langle\Psi|)^{-1}\right| \Psi_{0}\right\rangle=\frac{\left\langle\Psi\left|K^{-1}\right| \Psi_{0}\right\rangle}{1+\left\langle\Psi\left|K^{-1}\right| \Psi\right\rangle} .
$$

In particular, for the diagonal case $\left|\Psi_{0}\right\rangle=|\Psi\rangle$ we have

$$
\left\langle\Psi\left|(K+|\Psi\rangle\langle\Psi|)^{-1}\right| \Psi\right\rangle=1-\frac{1}{1+\left\langle\Psi\left|K^{-1}\right| \Psi\right\rangle} .
$$

These relations read in the energy (spectral) representation, where we assume that $K$ is diagonal,

$$
\left\langle\Psi\left|(K+|\Psi\rangle\langle\Psi|)^{-1}\right| \Psi_{0}\right\rangle=\sum_{j, j^{\prime}}\left\langle\Psi \mid E_{j}\right\rangle\left\langle E_{j}\left|(K+|\Psi\rangle\langle\Psi|)^{-1}\right| E_{j^{\prime}}\right\rangle\left\langle E_{j^{\prime}} \mid \Psi_{0}\right\rangle=\sum_{j, j^{\prime}}(K+\Pi E)_{E_{j}, E_{j^{\prime}}}^{-1}\left\langle\Psi \mid E_{j^{\prime}}\right\rangle\left\langle E_{j^{\prime}} \mid \Psi_{0}\right\rangle
$$


and

$$
\left\langle\Psi\left|(K+|\Psi\rangle\langle\Psi|)^{-1}\right| \Psi\right\rangle=\sum_{j, j^{\prime}}\left\langle\Psi \mid E_{j}\right\rangle\left\langle E_{j}\left|(K+|\Psi\rangle\langle\Psi|)^{-1}\right| E_{j^{\prime}}\right\rangle\left\langle E_{j^{\prime}} \mid \Psi\right\rangle=\sum_{j, j^{\prime}}(K+\Pi E)_{E_{j}, E_{j^{\prime}}}^{-1} \Pi_{E_{j^{\prime}}},
$$

where $K$ on the right-hand side is in the energy representation $\left\langle E_{j}|K| E_{j^{\prime}}\right\rangle=K_{E_{j}, E_{j}} \delta_{E_{j}, E_{j^{\prime}}}$. This implies with Eq. B3

$$
\sum_{j, j^{\prime}}(K+\Pi E)_{E_{j}, E_{j^{\prime}}}^{-1} \Pi_{E_{j^{\prime}}}=1-\frac{1}{1+\sum_{j} K_{E_{j}, E_{j}}^{-1} \Pi_{E_{j}}}
$$

and with Eq. (B2)

$$
\sum_{j, j^{\prime}}(K+\Pi E)_{E_{j}, E_{j^{\prime}}}^{-1}\left\langle\Psi \mid E_{j^{\prime}}\right\rangle\left\langle E_{j^{\prime}} \mid \Psi_{0}\right\rangle=\frac{\sum_{j} K_{E_{j}, E_{j}}^{-1}\left\langle\Psi \mid E_{j}\right\rangle\left\langle E_{j} \mid \Psi_{0}\right\rangle}{1+\sum_{j} K_{E_{j}, E_{j}}^{-1} \Pi_{E_{j}}} .
$$

Here $\left|\Psi_{0}\right\rangle$ can be any state, implying that the relation holds for any $Q_{E_{j^{\prime}}}=\left\langle\Psi \mid E_{j^{\prime}}\right\rangle h_{E_{j^{\prime}}}\left\langle E_{j^{\prime}} \mid \Psi\right\rangle=p_{j^{\prime}} h_{E_{j^{\prime}}}$ :

$$
\sum_{j, j^{\prime}}(K+\Pi E)_{E_{j}, E_{j^{\prime}}}^{-1} Q_{E_{j^{\prime}}}=\frac{\sum_{j} K_{E_{j}, E_{j}}^{-1} Q_{E_{j}}}{1+\sum_{j} K_{E_{j}, E_{j}}^{-1} \Pi_{E_{j}}} .
$$

\section{Appendix C: Product matrices}

With the properties of Eq. (16) we get for $k^{\prime} \geq k$

$$
\phi_{k}^{*} \phi_{k^{\prime}}=\operatorname{Tr}\left[D_{k}^{*}(\mathbf{1}-E \Pi) \cdots D_{2}^{*}(\mathbf{1}-E \Pi) D_{1}^{*} E \Pi\right] \operatorname{Tr}\left[D_{k^{\prime}}(\mathbf{1}-E \Pi) \cdots D_{2}(\mathbf{1}-E \Pi) D_{1} E \Pi\right]
$$

due to $\operatorname{Tr}\left(A_{1}\right) \operatorname{Tr}\left(A_{2}\right)=\operatorname{Tr}\left(A_{1} \times A_{2}\right)$

$$
\phi_{k}^{*} \phi_{k^{\prime}}=\operatorname{Tr}\left\{\left[D_{k}^{*}(\mathbf{1}-E \Pi) \cdots D_{2}^{*}(\mathbf{1}-E \Pi) D_{1}^{*} E \Pi\right] \times\left[D_{k^{\prime}}(\mathbf{1}-E \Pi) \cdots D_{2}(\mathbf{1}-E \Pi) D_{1} E \Pi\right]\right\}
$$

and due to $A_{1} B_{1} \times A_{2} B_{2}=\left[A_{1} \times A_{2}\right]\left[B_{1} \times B_{2}\right]$

$$
\begin{gathered}
\phi_{k}^{*} \phi_{k^{\prime}}=\operatorname{Tr}\left\{\left[\mathbf{1} \times D_{k^{\prime}}(\mathbf{1}-E \Pi) \cdots \mathbf{1} \times D_{k+1}(\mathbf{1}-E \Pi)\right]\right. \\
\left.\left[D_{k}^{*}(\mathbf{1}-E \Pi) \times D_{k}(\mathbf{1}-E \Pi) \cdots D_{2}^{*}(\mathbf{1}-E \Pi) \times D_{2}(\mathbf{1}-E \Pi)\right] D_{1}^{*} E \Pi \times D_{1} E \Pi\right\} .
\end{gathered}
$$

Averaging with respect to independent random times $\left\{\tau_{k}\right\}$ then gives

$$
\left\langle\phi_{k}^{*} \phi_{k^{\prime}}\right\rangle_{\tau}=\operatorname{Tr}\left\{\hat{C}_{2}^{k^{\prime}-k} \hat{\Gamma}^{k-1} \hat{G}\right\} \text { with } \hat{C}_{2}=\mathbf{1} \times\langle D\rangle_{\tau}(\mathbf{1}-E \Pi)
$$

since

$$
\left\langle D^{*}(\mathbf{1}-E \Pi) \times D(\mathbf{1}-E \Pi)\right\rangle_{\tau}=\left\langle D^{*} \times D\right\rangle_{\tau}(\mathbf{1}-E \Pi) \times(\mathbf{1}-E \Pi)
$$

from the first relation in Eq. (16) implies

$$
\begin{gathered}
\operatorname{Tr}\left\{\mathbf{1} \times\langle D\rangle_{\tau}(\mathbf{1}-E \Pi) \cdots \mathbf{1} \times\langle D\rangle_{\tau}(\mathbf{1}-E \Pi)\left\langle D^{*}(\mathbf{1}-E \Pi) \times D(\mathbf{1}-E \Pi)\right\rangle_{\tau} \cdots\left\langle D^{*}(\mathbf{1}-E \Pi) \times D(\mathbf{1}-E \Pi)\right\rangle_{\tau}\right. \\
\left.\left\langle D^{*} E \Pi \times D E \Pi\right\rangle_{\tau}\right\} \\
=\operatorname{Tr}\left\{\mathbf{1} \times\langle D\rangle_{\tau}(\mathbf{1}-E \Pi) \cdots \mathbf{1} \times\langle D\rangle_{\tau}(\mathbf{1}-E \Pi)\left\langle D^{*} \times D\right\rangle_{\tau}(\mathbf{1}-E \Pi) \times(\mathbf{1}-E \Pi) \cdots\left\langle D^{*} \times D\right\rangle_{\tau}(\mathbf{1}-E \Pi) \times(\mathbf{1}-E \Pi)\right. \\
\left.\left\langle D^{*} \times D\right\rangle_{\tau} E \Pi \times E \Pi\right\}=\operatorname{Tr}\left(\hat{C}_{2}^{k^{\prime}-k} \hat{\Gamma}^{k-1} \hat{G}\right),
\end{gathered}
$$

where the last equation follows from Eqs. (20), (21). An analog expression exists for $k \geq k^{\prime}$, such that we get

$$
\left\langle\phi_{k}^{*} \phi_{k^{\prime}}\right\rangle_{\tau}=\left\{\begin{array}{ll}
\operatorname{Tr}\left[\left(\hat{C}_{1}\right)^{k-k^{\prime}} \hat{\Gamma}^{k^{\prime}-1} \hat{G}\right] & \text { for } k \geq k^{\prime} \geq 1 \\
\operatorname{Tr}\left[\left(\hat{C}_{2}\right)^{k^{\prime}-k} \hat{\Gamma}^{k-1} \hat{G}\right] & \text { for } k^{\prime} \geq k \geq 1
\end{array} \quad, \quad \hat{C}_{j}=\left\{\begin{array}{ll}
\left\langle D^{*}\right\rangle_{\tau}(\mathbf{1}-E \Pi) \times \mathbf{1} & j=1 \\
\mathbf{1} \times\langle D\rangle_{\tau}(\mathbf{1}-E \Pi) & j=2
\end{array} .\right.\right.
$$




\section{Appendix D: Generating function}

Next we consider the Fourier transform of Eq. (C1)

$$
\begin{gathered}
\left\langle\tilde{\phi}^{*}(\omega) \tilde{\phi}\left(\omega+\omega^{\prime}\right)\right\rangle_{\tau}=\sum_{k \geq 1} \sum_{k^{\prime} \geq k} e^{i\left(\omega+\omega^{\prime}\right)\left(k^{\prime}-k\right)+i \omega^{\prime} k}\left\langle\phi_{k}^{*} \phi_{k^{\prime}}\right\rangle_{\tau}+\sum_{k^{\prime} \geq 1} \sum_{k>k^{\prime}} e^{i \omega\left(k^{\prime}-k\right)+i \omega^{\prime} k^{\prime}}\left\langle\phi_{k}^{*} \phi_{k^{\prime}}\right\rangle_{\tau} \\
=e^{i \omega^{\prime}} \operatorname{Tr}\left\{\left[\left(\hat{\mathbf{1}}-e^{i\left(\omega+\omega^{\prime}\right)} \hat{C}_{2}\right)^{-1}+e^{-i \omega} \hat{C}_{1}\left(\hat{\mathbf{1}}-e^{-i \omega} \hat{C}_{1}\right)^{-1}\right]\left(\hat{\mathbf{1}}-e^{i \omega^{\prime}} \hat{\Gamma}\right)^{-1} \hat{G}\right\},
\end{gathered}
$$

which becomes, after rewriting the second term in the trace,

$$
=e^{i \omega^{\prime}} \operatorname{Tr}\left\{\left[\left(\hat{\mathbf{1}}-e^{i\left(\omega+\omega^{\prime}\right)} \hat{C}_{2}\right)^{-1}+\left(\hat{\mathbf{1}}-e^{-i \omega} \hat{C}_{1}\right)^{-1}-\hat{\mathbf{1}}\right]\left(\hat{\mathbf{1}}-e^{i \omega^{\prime}} \hat{\Gamma}\right)^{-1} \hat{G}\right\}
$$

and with the expression of $F(\omega)$ in Eq. (23)

$$
=e^{i \omega^{\prime}} \operatorname{Tr}\left\{\left[\left(\hat{\mathbf{1}}-e^{i\left(\omega+\omega^{\prime}\right)} \hat{C}_{2}\right)^{-1}+\left(\hat{\mathbf{1}}-e^{-i \omega} \hat{C}_{1}\right)^{-1}\right]\left(\hat{\mathbf{1}}-e^{i \omega^{\prime}} \hat{\Gamma}\right)^{-1} \hat{G}\right\}-F\left(\omega^{\prime}\right) .
$$

To calculate the first term we can use the identity (B5). With $A_{1} \times\left(A_{2}+B_{2}\right)=A_{1} \times A_{2}+A_{1} \times B_{2}$ we get the relation

$$
\left(\hat{\mathbf{1}}-z \hat{C}_{j}\right)^{-1}= \begin{cases}{\left[\mathbf{1}-z\left\langle D^{*}\right\rangle_{\tau}(\mathbf{1}-E \Pi)\right]^{-1} \times \mathbf{1}} & j=1 \\ \mathbf{1} \times\left[\mathbf{1}-z\langle D\rangle_{\tau}(\mathbf{1}-E \Pi)\right]^{-1} & j=2\end{cases}
$$

such that

$$
\left.\left[\mathbf{1}-z\left\langle D^{*}\right\rangle_{\tau}(\mathbf{1}-E \Pi)\right]^{-1}=\left[\left(z\left\langle D^{*}\right\rangle_{\tau}\right)^{-1}-\mathbf{1}+E \Pi\right)\right]^{-1}\left(z\left\langle D^{*}\right\rangle_{\tau}\right)^{-1} .
$$

This yields for the first term in Eq. (D2)

$$
\begin{gathered}
\left.\operatorname{Tr}\left\{\left(\mathbf{1} \times\left[\mathbf{1}-e^{i\left(\omega+\omega^{\prime}\right)}\langle D\rangle_{\tau}(\mathbf{1}-E \Pi)\right]^{-1}\right)\left(\hat{\mathbf{1}}-e^{i \omega^{\prime}} \hat{\Gamma}\right)^{-1} \hat{G}\right)\right\} \\
\left.=\operatorname{Tr}\left\{\hat{\Pi}\left(\mathbf{1} \times\left[\mathbf{1}-e^{i\left(\omega+\omega^{\prime}\right)}\langle D\rangle_{\tau}(\mathbf{1}-E \Pi)\right]^{-1}\right)\left(\hat{\mathbf{1}}-e^{i \omega^{\prime}} \hat{\Gamma}\right)^{-1}\langle\hat{D}\rangle_{\tau} \hat{E}\right)\right\} \\
\left.=\operatorname{Tr}\left\{\left(\mathbf{1} \times\left[\mathbf{1}-e^{i\left(\omega+\omega^{\prime}\right)}\langle D\rangle_{\tau}(\mathbf{1}-\Pi E)\right]^{-1}\right) \hat{\Pi}\left(\hat{\mathbf{1}}-e^{i \omega^{\prime}} \hat{\Gamma}\right)^{-1}\langle\hat{D}\rangle_{\tau} \hat{E}\right)\right\} \\
=\sum_{j, j^{\prime}}\left[\mathbf{1}-e^{i\left(\omega+\omega^{\prime}\right)}\langle D\rangle_{\tau}(\mathbf{1}-\Pi E)\right]_{j, j^{\prime}}^{-1} p_{j^{\prime}} A_{j^{\prime}}\left(\omega^{\prime}\right)
\end{gathered}
$$

with

$$
A_{j^{\prime}}\left(\omega^{\prime}\right)=\sum_{j_{1}, j_{2}, j_{3}}\left[\left(\hat{\mathbf{1}}-e^{i \omega^{\prime}} \hat{\Gamma}\right)^{-1}\left\langle D^{*} \times D\right\rangle_{\tau}\right]_{j_{1} j^{\prime}, j_{2} j_{3}} p_{j_{1}}
$$

These components are linked to the generating function $F\left(\omega^{\prime}\right)$ in Eq. (23) through $F\left(\omega^{\prime}\right)=\sum_{j} p_{j} A_{j}\left(\omega^{\prime}\right)$. Now we write

$$
\left.\sum_{j, j^{\prime}}\left[\mathbf{1}-e^{i\left(\omega+\omega^{\prime}\right)}\langle D\rangle_{\tau}(\mathbf{1}-\Pi E)\right]_{j, j^{\prime}}^{-1} p_{j^{\prime}} A_{j^{\prime}}=\sum_{j, j^{\prime}}\left[\left(e^{i\left(\omega+\omega^{\prime}\right)}\langle D\rangle_{\tau}\right)^{-1}-\mathbf{1}+\Pi E\right)\right]_{j, j^{\prime}}^{-1} \frac{p_{j^{\prime}} A_{j^{\prime}}\left(\omega^{\prime}\right)}{\left.e^{i\left(\omega+\omega^{\prime}\right)[}[D\rangle_{\tau}\right]_{j^{\prime} j^{\prime}}}
$$

and with $K=\left(e^{i\left(\omega+\omega^{\prime}\right)}\left\langle D^{*}\right\rangle_{\tau}\right)^{-1}-\mathbf{1}$ we can apply Eq. (BB6)

$$
\left.=\sum_{j, j^{\prime}}[K+\Pi E)\right]_{j, j^{\prime}}^{-1} \frac{p_{j^{\prime}} A_{j^{\prime}}\left(\omega^{\prime}\right)}{e^{i\left(\omega+\omega^{\prime}\right)}\left[\langle D\rangle_{\tau}\right]_{j^{\prime} j^{\prime}}}=e^{-i\left(\omega+\omega^{\prime}\right)} \frac{\sum_{j} K_{j, j}^{-1} p_{j} A_{j}\left(\omega^{\prime}\right) / \gamma_{j}^{*}}{1+\sum_{j} K_{j, j}^{-1} p_{j}}=\frac{\sum_{j} p_{j} A_{j}\left(\omega^{\prime}\right) \prod_{k \neq j}\left(1-e^{i\left(\omega+\omega^{\prime}\right)} \gamma_{k}^{*}\right)}{\sum_{j^{\prime}} p_{j^{\prime}} \prod_{k \neq j^{\prime}}\left(1-e^{i\left(\omega+\omega^{\prime}\right)} \gamma_{k}^{*}\right)}
$$


The corresponding calculation yields for the second term in Eq. (D2):

$$
\left.\operatorname{Tr}\left\{\left(\left[\mathbf{1}-e^{-i \omega}\left\langle D^{*}\right\rangle_{\tau}(\mathbf{1}-E \Pi)\right]^{-1} \times \mathbf{1}\right)\left(\hat{\mathbf{1}}-e^{-i \omega^{\prime}} \hat{\Gamma}\right)^{-1} \hat{G}\right)\right\}=\sum_{j, j^{\prime}}\left[\mathbf{1}-e^{-i \omega}\left\langle D^{*}\right\rangle_{\tau}(\mathbf{1}-\Pi E)\right]_{j, j^{\prime}}^{-1} p_{j^{\prime}} B_{j^{\prime}}\left(\omega^{\prime}\right)
$$

and again with Eq. (B6)

$$
=\sum_{j, j^{\prime}}\left(K^{\prime}+\Pi E\right)_{j, j^{\prime}}^{-1} \frac{p_{j^{\prime}} B_{j^{\prime}}\left(\omega^{\prime}\right)}{e^{-i \omega}\left[\left\langle D^{*}\right\rangle_{\tau}\right]_{j^{\prime} j^{\prime}}}=e^{i \omega} \frac{\sum_{j} K_{j, j}^{\prime-1} p_{j} B_{j}\left(\omega^{\prime}\right) / \gamma_{j}}{1+\sum_{j} K_{j, j}^{\prime-1} p_{j}}=\frac{\sum_{j} p_{j} B_{j}\left(\omega^{\prime}\right) \prod_{k \neq j}\left(1-e^{-i \omega} \gamma_{k}\right)}{\sum_{j^{\prime}} p_{j^{\prime}} \prod_{k \neq j^{\prime}}\left(1-e^{-i \omega} \gamma_{k}\right)}
$$

with

$$
B_{j^{\prime}}\left(\omega^{\prime}\right)=\sum_{j_{1}, j_{2}, j_{3}}\left[\left(\hat{\mathbf{1}}-e^{-i \omega^{\prime}} \hat{\Gamma}\right)^{-1}\left\langle D^{*} \times D\right\rangle_{\tau}\right]_{j^{\prime} j_{1}, j_{2} j_{3}} p_{j_{1}}, \quad K^{\prime}=\left(e^{-i \omega}\langle D\rangle_{\tau}\right)^{-1}-\mathbf{1}
$$

and $\sum_{j} p_{j} B_{j}\left(\omega^{\prime}\right)=F\left(\omega^{\prime}\right)$. This allows us to write for the Fourier transform of Eq. (C1)

$$
\left\langle\tilde{\phi}^{*}(\omega) \tilde{\phi}\left(\omega+\omega^{\prime}\right)\right\rangle_{\tau}=e^{i \omega^{\prime}} \sum_{j_{1}, j_{2}} p_{j_{1}} p_{j_{2}}\left(h_{j_{2}}+h_{j_{1}}^{\prime}\right) \sum_{j_{1}^{\prime}, j_{2}^{\prime}}\left[\left(\hat{\mathbf{1}}-e^{i \omega^{\prime}} \hat{\Gamma}\right)^{-1}\langle\hat{D}\rangle_{\tau}\right]_{j_{1} j_{2}, j_{1}^{\prime} j_{2}^{\prime}}-F\left(\omega^{\prime}\right)
$$

with

$$
h_{j}=\frac{\prod_{k \neq j}\left(1-e^{i\left(\omega+\omega^{\prime}\right)} \gamma_{k}^{*}\right)}{\sum_{j^{\prime}} p_{j^{\prime}} \prod_{k \neq j^{\prime}}\left(1-e^{i\left(\omega+\omega^{\prime}\right)} \gamma_{k}^{*}\right)}, \quad h_{j}^{\prime}=\frac{\prod_{k \neq j}\left(1-e^{-i \omega} \gamma_{k}\right)}{\sum_{j^{\prime}} p_{j^{\prime}} \prod_{k \neq j^{\prime}}\left(1-e^{-i \omega} \gamma_{k}\right)}, \quad \gamma_{k}=\left\langle e^{i E_{k} \tau}\right\rangle_{\tau} .
$$

These are analytic functions in $e^{i\left(\omega+\omega^{\prime}\right)}$ and $e^{-i \omega}$, respectively, and their special form implies $\sum_{j} p_{j} h_{j}=$ $\sum_{j} p_{j} h_{j}^{\prime}=1$. For $\omega^{\prime}=0$ the normalization $\left\langle|\tilde{\phi}(\omega)|^{2}\right\rangle_{\tau}=1$ can be obtained from Eq. (D6) with the help of Eq. (31). This can be shown by the following reasoning. From App. $\mathrm{A}$ we have $F\left(\omega^{\prime}=0\right)=1$. Then we can write with Eq. (D6)

$$
\left\langle|\tilde{\phi}(\omega)|^{2}\right\rangle_{\tau}=\sum_{j_{1}, j_{2}} p_{j_{1}} p_{j_{2}}\left(h_{j_{2}}+h_{j_{1}}^{\prime}\right) \sum_{j_{3}, j_{4}}\left[(\hat{\mathbf{1}}-\hat{\Gamma})^{-1}\langle\hat{D}\rangle_{\tau}\right]_{j_{1} j_{2}, j_{3} j_{4}}-1=\sum_{j_{1}, j_{2}} p_{j_{1}} p_{j_{2}}\left(h_{j_{2}}+h_{j_{1}}^{\prime}\right) T_{j_{1} j_{2}}-1 .
$$

Inserting now $T_{j_{1} j_{2}}$ from Eq. (31) and use $\sum_{j} p_{j} h_{j}=\sum_{j} p_{j} h_{j}^{\prime}=1$ we obtain 1 for this expression.

\section{Appendix E: Analytic properties of $F(\omega)$}

When we consider the trace term in Eq. (19) as

$$
\left\langle\left|\phi_{n+1}\right|^{2}\right\rangle_{\tau}=\operatorname{Tr}\left[(\hat{D} \hat{C})^{n} \hat{D} \hat{E} \hat{\Pi}\right]
$$

with the short-hand notation $\hat{D}=\langle\hat{D}\rangle_{\tau}$, we get in the Zeno limit $\hat{D} \rightarrow \hat{\mathbf{1}}$ a vanishing expression except for $n=0$, since $\hat{E} \hat{\Pi}$ and $\hat{C}$ are projectors with

$$
(\hat{E} \hat{\Pi})^{2}=\hat{E} \hat{\Pi}, \quad \hat{C}^{2}=\hat{C}, \quad \hat{E} \hat{\Pi} \hat{C}=\hat{E} \hat{\Pi}(\mathbf{1}-E \Pi) \times(\mathbf{1}-E \Pi)=0,
$$

and since for $n=0$

$$
\operatorname{Tr}(\hat{E} \hat{\Pi})=\sum_{j, j^{\prime}=1}^{N} p_{j} p_{j^{\prime}}=1
$$

Returning to Eq. (E1), we can write with $\hat{C}^{2}=\hat{C}$ and $\hat{R}=\hat{\mathbf{1}}-\hat{D}$

$$
(\hat{D} \hat{C})^{n}=\hat{D}(\hat{C} \hat{D} \hat{C})^{n}=\hat{D}[\hat{C}(\hat{\mathbf{1}}-\hat{R}) \hat{C}]^{n}=\hat{D}(\hat{C}-\hat{C} \hat{R} \hat{C})^{n}=\hat{D} \hat{C}(\hat{\mathbf{1}}-\hat{R} \hat{C})^{n}
$$


such that for $n \geq 1$

$$
\left\langle\left|\phi_{n+1}\right|^{2}\right\rangle_{\tau}=\operatorname{Tr}\left[\hat{C}(\hat{\mathbf{1}}-\hat{R} \hat{C})^{n} \hat{D} \hat{E} \hat{\Pi} \hat{D}\right]=\operatorname{Tr}\left[\hat{C}(\hat{\mathbf{1}}-\hat{R} \hat{C})^{n}(\hat{\mathbf{1}}-\hat{R}) \hat{E} \hat{\Pi}(\hat{\mathbf{1}}-\hat{R})\right]
$$

and with $\hat{E} \hat{\Pi} \hat{C}=\hat{C} \hat{E} \hat{\Pi}=0$

$$
\left\langle\left|\phi_{n+1}\right|^{2}\right\rangle_{\tau}=\operatorname{Tr}\left[\hat{C}(\hat{\mathbf{1}}-\hat{R} \hat{C})^{n} \hat{R} \hat{E} \hat{\Pi} \hat{R}\right]=\operatorname{Tr}\left[\hat{R} \hat{C}(\hat{\mathbf{1}}-\hat{R} \hat{C})^{n} \hat{R} \hat{E} \hat{\Pi}\right] .
$$

Moreover, with $\hat{R} \hat{C}=\hat{\mathbf{1}}-(\hat{\mathbf{1}}-\hat{R} \hat{C})$ we get

$$
\left\langle\left|\phi_{n+1}\right|^{2}\right\rangle_{\tau}=\operatorname{Tr}\left[(\hat{\mathbf{1}}-\hat{R} \hat{C})^{n} \hat{R} \hat{E} \hat{\Pi}\right]-\operatorname{Tr}\left[(\hat{\mathbf{1}}-\hat{R} \hat{C})^{n+1} \hat{R} \hat{E} \hat{\Pi}\right] .
$$

Now we introduce the projector $\hat{P}$ with $\hat{R}=\hat{P} \hat{R}$. Then we can write

$$
\hat{C}(\hat{\mathbf{1}}-\hat{R} \hat{C})^{n} \hat{R}=\hat{C}(\hat{\mathbf{1}}-\hat{R} \hat{C} \hat{P})^{n} \hat{R}=\hat{C}(\hat{\mathbf{1}}-\hat{P}+\hat{P}-\hat{R} \hat{C} \hat{P})^{n} \hat{R}=\hat{C}\left[(\hat{\mathbf{1}}-\hat{P})^{n}+(\hat{P}-\hat{R} \hat{C} \hat{P})^{n}\right] \hat{R}
$$

and since $(\hat{\mathbf{1}}-\hat{P})^{n} \hat{R}=0$

$$
=\hat{C}(\hat{P}-\hat{R} \hat{C} \hat{P})^{n} \hat{R} \text {. }
$$

The eigenvalues of $\hat{R} \hat{C} \hat{P}$ might be complex. Therefore, it is better to calculate the eigenvalues of the Hermitean matrix

$$
(\hat{R} \hat{C} \hat{P})^{\dagger} \hat{R} \hat{C} \hat{P}=\hat{P} \hat{C} \hat{R}^{\dagger} \hat{R} \hat{C} \hat{P}
$$

whose determinant reads

$$
\operatorname{det}\left(\hat{P} \hat{C} \hat{R}^{\dagger} \hat{R} \hat{C} \hat{P}\right)=\operatorname{det}(\hat{P} \hat{C} \hat{P})^{2} \prod_{j, j^{\prime}=1 ; j^{\prime} \neq j}^{N}\left|1-\left\langle D_{j j^{\prime}}\right\rangle_{\tau}\right|^{2} .
$$

A necessary condition for a quick decay of $(\hat{P}-\hat{R} \hat{C} \hat{P})^{n}$ with $n$ is that the product of $\left|1-\left\langle D_{j j^{\prime}}\right\rangle_{\tau}\right|^{2}$ is not small, while the sufficient condition requires that $\operatorname{det}(\hat{P} \hat{C} \hat{P})$ also is not small. To see the latter, we analyze the elements of the projected matrix

$$
(\hat{P} \hat{C} \hat{P})_{j j^{\prime}, k k^{\prime}}=\left(1-\delta_{j j^{\prime}}\right)\left(1-\delta_{k k^{\prime}}\right)\left(\delta_{j k}-p_{k}\right)\left(\delta_{j^{\prime} k^{\prime}}-p_{k^{\prime}}\right) .
$$

We only consider the projected matrix, which has the following matrix elements with $j \neq j^{\prime}$ and $k \neq k^{\prime}$ :

$$
k=j, k^{\prime}=j^{\prime}:(\hat{P} \hat{C} \hat{P})_{j j^{\prime}, j j^{\prime}}=\left(1-p_{j}\right)\left(1-p_{j^{\prime}}\right), \quad k \neq j, k^{\prime} \neq j^{\prime}: \quad(\hat{P} \hat{C} \hat{P})_{j j^{\prime}, k k^{\prime}}=p_{k} p_{k^{\prime}}
$$

and

$$
k=j, k^{\prime} \neq j^{\prime}: \quad(\hat{P} \hat{C} \hat{P})_{j j^{\prime}, j k^{\prime}}=-\left(1-p_{j}\right) p_{k^{\prime}}, \quad k \neq j, k^{\prime}=j^{\prime}: \quad(\hat{P} \hat{C} \hat{P})_{j j^{\prime}, k j^{\prime}}=-p_{k}\left(1-p_{j^{\prime}}\right) .
$$

For the special case of $N=2$ this gives a $2 \times 2$ matrix:

$$
\left(\begin{array}{cc}
0 & -p_{2}\left(1-p_{2}\right) \\
-p_{1}\left(1-p_{1}\right) & 0
\end{array}\right)
$$

whose determinant $-p_{1}\left(1-p_{1}\right) p_{2}\left(1-p_{2}\right)$ vanishes only for $p_{1}=0,1$ and/or $p_{2}=0,1$.

\section{Appendix F: Symmetric two-level system}

The matrix structure of the symmetric $2 \mathrm{LS}$ reads

$$
\left([A \times B]_{i j, k l}\right)=\left(A_{i k} B_{j l}\right)=\left(\begin{array}{cccc}
A_{11} B_{11} & A_{11} B_{12} & A_{12} B_{11} & A_{12} B_{12} \\
A_{11} B_{21} & A_{11} B_{22} & A_{12} B_{21} & A_{12} B_{22} \\
A_{21} B_{11} & A_{21} B_{12} & A_{22} B_{11} & A_{22} B_{12} \\
A_{21} B_{21} & A_{21} B_{22} & A_{22} B_{21} & A_{22} B_{22}
\end{array}\right)
$$


and

$$
\left(\Gamma_{i j, k l}\right)=\left(\begin{array}{llll}
\Gamma_{11,11} & \Gamma_{11,12} & \Gamma_{11,21} & \Gamma_{11,22} \\
\Gamma_{12,11} & \Gamma_{12,12} & \Gamma_{12,21} & \Gamma_{12,22} \\
\Gamma_{21,11} & \Gamma_{21,12} & \Gamma_{21,21} & \Gamma_{21,22} \\
\Gamma_{22,11} & \Gamma_{22,12} & \Gamma_{22,21} & \Gamma_{22,22}
\end{array}\right)
$$

Then we have

$$
\left\langle D^{*} \times D\right\rangle_{\tau}=\left(\begin{array}{cccc}
1 & 0 & 0 & 0 \\
0 & \left\langle e^{2 i J \tau}\right\rangle_{\tau} & 0 & 0 \\
0 & 0 & \left\langle e^{-2 i J \tau}\right\rangle_{\tau} & 0 \\
0 & 0 & 0 & 1
\end{array}\right)=\left(\begin{array}{cccc}
1 & 0 & 0 & 0 \\
0 & y & 0 & 0 \\
0 & 0 & y^{*} & 0 \\
0 & 0 & 0 & 1
\end{array}\right)
$$

with $y=\left\langle e^{2 i J \tau}\right\rangle_{\tau}$. Moreover,

$$
(\mathbf{1}-E \Pi) \times(\mathbf{1}-E \Pi)=\frac{1}{4}\left(\begin{array}{cccc}
1 & -1 & -1 & 1 \\
-1 & 1 & 1 & -1 \\
-1 & 1 & 1 & -1 \\
1 & -1 & -1 & 1
\end{array}\right)
$$

such that

$$
\hat{\Gamma}=\left\langle D^{*} \times D\right\rangle_{\tau}(\mathbf{1}-E \Pi) \times(\mathbf{1}-E \Pi)=\frac{1}{4}\left(\begin{array}{cccc}
-1 & 1 & 1 & -1 \\
y & -y & -y & y \\
y^{*} & -y^{*} & -y^{*} & y^{*} \\
-1 & 1 & 1 & -1
\end{array}\right)
$$

with three vanishing eigenvalues and one eigenvalue $\left(y+y^{*}+2\right) / 4$. With the help of Maxima we obtain with $c=z / 4$

$$
\begin{aligned}
& \operatorname{det}(\hat{\mathbf{1}}-z \hat{\Gamma})=1-c\left(2+y+y^{*}\right)=1-\frac{z}{2}\left[1+\langle\cos (2 J \tau)\rangle_{\tau}\right]=1-z\left\langle\cos ^{2}(J \tau)\right\rangle_{\tau} \\
& (\hat{\mathbf{1}}-z \hat{\Gamma})^{-1}=\frac{-1}{1-z\left\langle\cos ^{2}(J \tau)\right\rangle_{\tau}}\left(\begin{array}{cccc}
c\left(y+y^{*}+1\right)-1 & c & c & -c \\
c y & c\left(y^{*}+2\right)-1 & -c y & c y \\
c y^{*} & -c y^{*} & c(y+2)-1 & c y^{*} \\
-c & c & c & c\left(y+y^{*}+1\right)-1
\end{array}\right) \\
& (\hat{\mathbf{1}}-z \hat{\Gamma})^{-1}\left\langle D^{*} \times D\right\rangle_{\tau} \\
& =\frac{-1}{1-z\left\langle\cos ^{2}(J \tau)\right\rangle_{\tau}}\left(\begin{array}{cccc}
c\left(y+y^{*}+1\right)-1 & c y & c y^{*} & -c \\
c y & c\left(y y^{*}+2 y\right)-y & -c y y^{*} & c y \\
c y^{*} & -c y y^{*} & c\left(y y^{*}+2 y^{*}\right)-y^{*} & c y^{*} \\
-c & c y & c y^{*} & c\left(y+y^{*}+1\right)-1
\end{array}\right)
\end{aligned}
$$

Then the generating function $F(\omega)$ in Eq. (23) reads

$$
F(\omega)=\frac{4 e^{2 i \omega}\langle\cos 2 J \tau\rangle_{\tau}-2 e^{i \omega}\left(\langle\cos 2 J \tau\rangle_{\tau}+1\right)}{2 e^{i \omega}\left(\langle\cos 2 J \tau\rangle_{\tau}+1\right)-4},
$$

which gives for $\omega=0$

$$
F(0)=1, \quad-i F^{\prime}(0)=2, \quad-F^{\prime \prime}(0)=2 \frac{3-\langle\cos 2 J \tau\rangle_{\tau}}{1-\langle\cos 2 J \tau\rangle_{\tau}} .
$$

For the $\phi$-correlator we get the winding number

$$
w_{\phi}=\left.\frac{1}{2 \pi i} \int_{0}^{2 \pi} \partial_{\omega^{\prime}} \log \left(\left\langle\tilde{\phi}^{*}(\omega) \tilde{\phi}\left(\omega+\omega^{\prime}\right)\right\rangle_{\tau}\right)\right|_{\omega^{\prime}=0} d \omega=\frac{1}{2 \pi i} \int \frac{a_{2} z^{2}+a_{1} z+a_{0}}{4\left(y+y^{*}-2\right)(z-C)(C z-1)} \frac{1}{z} d z
$$


with $C=\langle\cos J \tau\rangle_{\tau}$ and $y=\left\langle e^{2 i J \tau}\right\rangle_{\tau}$, with poles

$$
z_{0}=0, z_{1}=C, z_{2}=1 / C
$$

and with the coefficients

$$
\begin{gathered}
a_{0}=a_{2}=\left(6 x^{*}+2 x\right) y^{*}+\left(2 x^{*}+6 x\right) y-8 x^{*}-8 x \\
a_{1}=4\left[\left(-x^{* 2}-x x^{*}-2\right) y^{*}+\left(-x x^{*}-x^{2}-2\right) y+x^{* 2}+2 x x^{*}+x^{2}+4\right],
\end{gathered}
$$

where $x=\left\langle e^{-i J \tau}\right\rangle_{\tau}$. After performing the Cauchy integration in Eq. (F1) for the two poles $z_{0,1}$ we get

$$
w_{\phi}=2 .
$$

[1] M. Štefaňák, I. Jex, and T. Kiss, Recurrence and pólya number of quantum walks, Phys. Rev. Lett. 100, 020501 (2008)

[2] E. Bach, S. Coppersmith, M. P. Goldschen, R. Joynt, and J. Watrous, One-dimensional quantum walks with absorbing boundaries, Journal of Computer and System Sciences 69, 562 (2004).

[3] H. Friedman, D. A. Kessler, and E. Barkai, Quantum walks: The first detected passage time problem, Phys. Rev. E 95, 032141 (2017)

[4] S. Dhar, S. Dasgupta, A. Dhar, and D. Sen, Detection of a quantum particle on a lattice under repeated projective measurements, Phys. Rev. A 91, 062115 (2015)

[5] S. Dhar, S. Dasgupta, and A. Dhar, Quantum time of arrival distribution in a simple lattice model, Journal of Physics A: Mathematical and Theoretical 48, 115304 (2015).

[6] H. Krovi and T. A. Brun, Quantum walks with infinite hitting times, Phys. Rev. A 74, 042334 (2006)

[7] H. Krovi and T. A. Brun, Hitting time for quantum walks on the hypercube, Phys. Rev. A 73, 032341 (2006)

[8] H. Krovi and T. A. Brun, Quantum walks on quotient graphs, Phys. Rev. A 75, 062332 (2007)

[9] F. A. Grünbaum, L. Velázquez, A. H. Werner, and R. F. Werner, Recurrence for discrete time unitary evolutions, Communications in Mathematical Physics 320, 543 (2013).

[10] P. Krapivsky, J. Luck, and K. Mallick, Survival of classical and quantum particles in the presence of traps, J Stat Phys 154, 1430 (2014).

[11] J. Bourgain, F. A. Grünbaum, L. Velázquez, and J. Wilkening, Quantum recurrence of a subspace and operator-valued schur functions, Communications in Mathematical Physics 329, 1031 (2014).

[12] P. Sinkovicz, T. Kiss, and J. K. Asbóth, Generalized kac lemma for recurrence time in iterated open quantum systems, Phys. Rev. A 93, 050101 (2016)

[13] H. Friedman, D. A. Kessler, and E. Barkai, Quantum renewal equation for the first detection time of a quantum walk, Journal of Physics A: Mathematical and Theoretical 50, 04LT01 (2016).

[14] F. Thiel, E. Barkai, and D. A. Kessler, First detected arrival of a quantum walker on an infinite line, Phys. Rev. Lett. 120, 040502 (2018)

[15] T. Nitsche, S. Barkhofen, R. Kruse, L. Sansoni, M. Štefaňák, A. Gábris, V. Potoček, T. Kiss, I. Jex, and C. Silberhorn, Probing measurement-induced effects in quantum walks via recurrence, Science Advances 4, 10.1126/sciadv.aar6444 (2018), https://advances.sciencemag.org/content/4/6/eaar6444.full.pdf

[16] P. L. Krapivsky, J. M. Luck, and K. Mallick, Quantum return probability of a system of N non-interacting lattice fermions, Journal of Statistical Mechanics: Theory and Experiment 2, 023104 (2018) arXiv:1710.08178 [cond-mat.mes-hall]

[17] S. Lahiri and A. Dhar, Return to the origin problem for a particle on a one-dimensional lattice with quasi-zeno dynamics, Phys. Rev. A 99, 012101 (2019)

[18] R. Yin, K. Ziegler, F. Thiel, and E. Barkai, Large fluctuations of the first detected quantum return time, Phys. Rev. Research 1, 033086 (2019).

[19] V. Dubey, C. Bernardin, and A. Dhar, Quantum Dynamics under continuous projective measurements: non-Hermitian description and the continuous space space limit, arXiv e-prints , arXiv:2012.01196 (2020), arXiv:2012.01196 [quant-ph]

[20] Q. Liu, R. Yin, K. Ziegler, and E. Barkai, Quantum walks: The mean first detected transition time, Phys. Rev. Research 2, 033113 (2020)

[21] M. Varbanov, H. Krovi, and T. A. Brun, Hitting time for the continuous quantum walk, Phys. Rev. A 78, 022324 (2008).

[22] A. Riera-Campeny, J. Ollé, and A. Masó-Puigdellosas, Measurement-induced resetting in open quantum systems, arXiv e-prints , arXiv:2011.04403 (2020), arXiv:2011.04403 [quant-ph] 
[23] S. Redner, A Guide to First-Passage Processes (Cambridge University Press, 2001).

[24] O. Bénichou, T. Guérin, and R. Voituriez, Mean first-passage times in confined media: from markovian to non-markovian processes, Journal of Physics A: Mathematical and Theoretical 48, 163001 (2015).

[25] E. J. Heller, Quantum localization and the rate of exploration of phase space, Phys. Rev. A 35, 1360 (1987)

[26] D. Cohen, V. I. Yukalov, and K. Ziegler, Hilbert-space localization in closed quantum systems, Phys. Rev. A 93, 042101 (2016).

$[27]$ M. V. Berry, Quantal Phase Factors Accompanying Adiabatic Changes, Proceedings of the Royal Society of London Series A 392, 45 (1984) 